Review

\title{
Metals on Graphene: Interactions, Growth Morphology, and Thermal Stability
}

\author{
Xiaojie Liu ${ }^{1,2}$, Cai-Zhuang Wang ${ }^{2, *}$, Myron Hupalo ${ }^{2}$, Hai-Qing Lin ${ }^{1}$, Kai-Ming Ho ${ }^{2}$ and \\ Michael C. Tringides ${ }^{2}$
}

1 Beijing Computational Science Research Center, Beijing 100084, China;

E-Mails: xiaojie@csrc.ac.cn (X.L.); haiqing0@csrc.ac.cn (H.-Q.L.)

2 Ames Laboratory-US Department of Energy, Department of Physics and Astronomy, Iowa State University, Ames, IA 50011, USA; E-Mails: hupalo@ameslab.gov (M.H.);

kmh@ameslab.gov (K.-M.H.); tringides@ameslab.gov (M.C.T.)

* Author to whom correspondence should be addressed; E-Mail: wangcz@ameslab.gov;

Tel.: +1-515-294-6934; Fax: +1-515-294-0689.

Received: 30 November 2012; in revised form: 14 January 2013 / Accepted: 24 January 2013 / Published: 31 January 2013

\begin{abstract}
Graphene, a single atomic layer of graphite, has been a material of recent intensive studies due to its novel electronic and structural properties and its potential applications in the emerging area of carbon-based electronic devices. Metal on graphene growth is one of the current research interests, aiming at improving and manipulating the electronic and magnetic properties of graphene through metal atom adsorption or doping to meet various requirements in device applications. In this paper, we will give an overview of recent experimental and computational investigation of interaction, growth morphology, and thermal stability of various metals on graphene grown on $6 \mathrm{H}-\mathrm{SiC}(0001)$ substrate.
\end{abstract}

Keywords: metal on graphene; adsorption; scanning tunneling microscopy; interaction; growth morphology; thermal stability

\section{Introduction}

Graphene, a planar monolayer of carbon atoms arranged into a two-dimensional (2D) honeycomb lattice, has attracted tremendous interest in physics, chemistry, and material science since 2004 [1]. Graphene exhibits extraordinary electronic structure: Its $\pi$ and $\pi^{*}$ bands merge together into a single 
point at the Fermi energy the so-called Dirac point and display a linear E vs. k dispersion. Such a unique feature implies that the electrons at the Fermi level in graphene behave like mass-less relativistic particles and lead to some unusual properties such as the anomalous quantum Hall effect [2,3], high electron mobility at room temperature [1,3], and exceptional thermal [4] and mechanical [5] properties. There is no doubt that materials based on graphene will have great potentials in electronic devices, nanotechnology, surface catalysis, data storage, and magnetic memory applications [6-9].

In addition to the intrinsic properties of pure graphene, a lot of recent experimental and theoretical studies [10-64] have been devoted to the investigations of metals on graphene, aiming at improving and manipulating the electronic and magnetic properties of graphene through metal atom adsorption or doping to meet various requirements in device applications [6-9]. For example, recent studies [10-14,31-37] showed that alkali adatoms adsorption on graphene release their valence electron easily and act as good agents for $n$-doping of graphene. In addition to alkali metal adsorptions, research interest in transition and noble metal elements [38-49,53] or clusters [50-52,54-57] adsorbed on graphene continues to grow rapidly and broadens the range of applications from catalysis [65-70], spintronics [9,27,28], to nanomagnetic devices [26,27,38-49]. Transition and noble metal nanostructures on graphene have been shown to be efficient as supported catalysis [22,24,25,65-70]. Another technological interest is to grow uniform magnetic metal films for use as spin filters in spintronics or to grow high density of magnetic islands that are thermally stable for computer storage applications. Moreover, the study of metals on graphene is also important for a better understanding of the quality of metal contacts which is very critical to the performance of graphene based microelectronics devices. On the other hands, impurities on graphene [58-62] also induce large magnetism or form strong covalent bonds to modify the electronic and magnetic properties of pure graphene to meet the needs of various graphene-based materials and device applications. In this paper, we will give an overview on recent experimental and theoretical investigations to understand the growth morphology and thermal stability of metal nanostructures and thin film grown on grapheme [71-78]. We note diverse efforts have been made to synthesize graphene with different approaches, such as exfoliation and cleavage from graphite [1], chemical vapor deposition on metal surfaces [79-88], carbon deposition on single crystal metal surfaces under ultra high vacuum (UHV) conditions [83,89] and thermal annealing of $\mathrm{SiC}$ [90-95]. It is not the scope of this paper to review the growth and preparation of graphene. We refer the readers to several excellent review articles [82,83] on the current state of graphene growth. Our emphasis is the growth of metals on graphene. We also note that in the literature, growth of various metals on graphene with different substrate (e.g., on Si-terminated $\mathrm{SiC}, \mathrm{SiO}_{2}$, or on transition metal surfaces) have been reported, but with the growth outcome depending on the type of graphene and the substrate is grown on. For example, for graphene grown on metals, the moiré pattern formed between graphene and the metal substrate on $\mathrm{Cu}(111)$ [86], $\mathrm{Ni}(111)$ [85,87], $\mathrm{Au}(111)$ [88], $\mathrm{Ru}(0001)$ [22,25,30], and $\operatorname{Ir}(111)$ [27-29,79-81] have been considered as a good substrate for patterned growth of metal clusters networks. The reason for this is because the graphene substrate interaction is strong so the moiré supercell provides the nucleation sites for the deposited atoms to aggregate regular island distributions. For example, by using STM, Goodman et al. reported the growth morphology and properties of $\mathrm{Pt}, \mathrm{Rh}, \mathrm{Pd}, \mathrm{Co}$, and $\mathrm{Au}$ islands on graphene supported on the template of a graphene moiré pattern formed on $\mathrm{Ru}(0001)$ substrate [17,22,25]. 
Recently, Ir, Pt, W, Re, Co, Eu, Au, AuIr, and FeIr nanoislands have been grown on the graphene moiré structure on $\operatorname{Ir}(111)$ substrate as shown by Michely et al. [16,27-29,79-81]. Noble metal clusters on graphene prepared by chemical methods have also been shown to enhance the electrocatalytic activity [65-70].

This review article will focus only on epitaxial growth of metal on graphene prepared by thermal annealing of SiC. This is an area where joint experimental and theoretical studies to relate measured nanostructure morphologies and calculated metal-graphene interactions are limited. In addition, graphene on Si-terminated $\mathrm{SiC}$ behaves differently from the graphene on transition metal substrate [79-88] with regards to metal nanostructure formation morphology and stability. The interaction between the graphene and Si-terminated $\mathrm{SiC}$ substrate is believed to be weak and the substrate does not play an important role in controlling the growth morphology of the metal islands on graphene. The growth morphology of metal islands is therefore dominated by the interaction between the metal adatoms and graphene. Only limited experimental work has been done in this area with the main goal to intercalate the metal at the graphene-SiC interface, as shown in recently study [96], to induce superconductivity [97], to study potassium diffusion [14], to fully decouple graphene from the substrate [98], to modify the electronic structure with Li [11], as well as to study different types of intercalation with sodium [99]. The combined experimental and theoretical studies presented in this review have focused over a wide temperature range; but prior to intercalation, so the changing morphology can be monitored while the metal atoms are still on the surface and have not diffused at the interface.

The remaining of the paper is arranged as follows. In Section 2, we will review the experimental observation of growth morphology of various metals on graphene using scanning tunneling microscopy (STM). Section 3 will focus on the interaction between the metal adatoms and graphene from first-principles calculations, aiming at providing a microscopic understanding of the growth morphology of various metals on graphene observed by experiment. Microscopic bonding mechanism between metal adatoms and graphene and the changes in the electronic structures of graphene induced by adsorption of metal adatoms will be reviewed in more details in Sections 3 and 4. The correlations between the adsorption behaviors from the first-principles calculations and growth morphology observed in experiment will be further discussed in Section 5. In Section 6, we will review our current understanding of interaction between metal adatoms graphene and their effects on the growth morphology. A discussion of thermal stability of nanostructures on graphene will be given in Section 7. Finally, some concluding remarks will be given in Section 8.

\section{Growth Morphology of Metal on Graphene}

Experimental studies of growth morphology of metal on graphene on Si-terminated SiC substrate have been carried out recently [71-78]. The graphene layer was prepared by the thermal annealing of $\mathrm{SiC}$ [91]. As shown in Figure 1, the method produces G1 (single layer) or G2 (bilayer) graphene of very high quality [91] with domains that extend over $\mu \mathrm{ms}$ because of slow and controlled desorption of Si through the steps to graphitize the surface. The steps undergo a single to triple step transition with different retracting speeds that results in the fine coverage control of the remaining $\mathrm{C}$ that eventually produces uniform graphene layers over very long distances [91]. 
Figure 1. (a) $250 \times 250 \mathrm{~nm}^{2}$ area scanning tunneling microscopy (STM) image showing different heights on a graphitized $6 \mathrm{H}-\mathrm{SiC}(0001)$ sample; (b) The outlined white box of Figure 1a. All step heights can be written in terms of $\mathrm{s}=0.25 \mathrm{~nm}$ or $\mathrm{g}=0.35 \mathrm{~nm}$; (c) Single layer graphene growing uninterrupted over a graphene step.

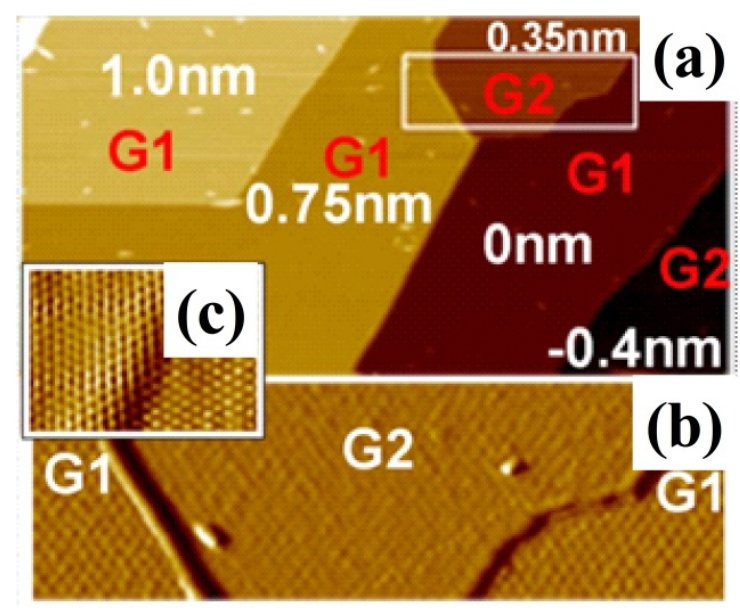

Epitaxial growth of several metals ( $\mathrm{Gd}, \mathrm{Dy}, \mathrm{Eu}, \mathrm{Fe}$, and $\mathrm{Pb}$ ) has been performed experimentally. The growth morphology, such as the island dimensionality (i.e., two-dimensional (2D) or three-dimensional (3D)), island shape and island density at various deposition rates and coverages, as well as deposition temperature has been measured using scanning tunneling microscopy (STM). STM is a useful tool to study metal graphene interaction because the grown island morphology under different conditions can reveal information about the energetic barriers of metal adsorption and diffusion on graphene. Using STM, one can observe directly whether islands or films are grown at given set of growth conditions and whether they are crystalline or amorphous. STM can also be used to measure the nucleated island density $n$ to deduce the energetic and kinetic parameters to compare with the ones calculated using the first-principles methods as will be discussed in the next section. The island density can be analyzed in terms of the classical theory of nucleation [100], which has been extensively used in STM experiments to deduce the diffusion barrier $\Delta E$ in numerous metal $/ \mathrm{metal}$ and metal/semiconductor systems [100]. Another quantity easily measurable by STM is whether growth is smooth (layer-by-layer) or rough three dimensional. The difference in morphology is controlled both by surface diffusion and the step edge barrier, i.e., an extra barrier existing at step edges $\Delta E_{\mathrm{s}}$ that controls how easy the atoms move from higher to lower layers. This barrier especially in the first layer is defined by the adatom and substrate, so it also contains information about the strength of the metal-graphene interaction. Small $\Delta E$ and $\Delta E_{\mathrm{s}}$ imply layer-by-layer growth while in the opposite limit the growth is three dimensional. Both these barriers indirectly reveal information about the strength of the metal-graphene interaction. As one can see in the following, different metals on graphene exhibit very different growth morphologies, indicating different interaction strength between the metals and graphene and even among the metal adatoms. 


\subsection{Gd on Graphene}

Figure 2 shows Gd deposition at room temperature (RT) as a function of coverage $\theta$ with the same flux rate $\mathrm{F}=0.12$ monolayer (ML)/min. Fractal-like Gd 3D islands have been seen for all coverages. While the island size increases with coverage, the island density $n$ is constant at approximately the value of 0.0014 islands $/ \mathrm{nm}^{2}$ over a wide range of coverage $\theta$ as one can see from Figure 2. The independence of the island density on $\theta$ is consistent with the predictions of the scaling theory of nucleation [101]. With increasing $\theta$ each fractal island collects the deposited atoms and grows with the arm widths getting wider. The island volume normalized to the same $250 \times 250 \mathrm{~nm}^{2}$ area increases from average film height 0.29 to $1.05 \mathrm{~nm}$ in Figure 2c,d respectively which approximately scales with the deposition times $10 \mathrm{~min}$ in Figure $2 \mathrm{c}$ and $30 \mathrm{~min}$ in Figure $2 \mathrm{~d}$. This scaling also is another indication that is no intercalation of Gd below graphene.

Figure 2. STM image of $\mathrm{Gd}$ on graphene (a) $210 \times 210 \mathrm{~nm}^{2}, \theta=0.5 \mathrm{ML}$; (b) $250 \times 250 \mathrm{~nm}^{2}, \theta=1.2 \mathrm{ML}$; (c) $250 \times 250 \mathrm{~nm}^{2}, \theta=3.6 \mathrm{ML}$; (d) Constant island density vs. coverage at room temperature (RT) for Gd on graphene with $\mathrm{F}=0.12 \mathrm{ML} / \mathrm{min}$.
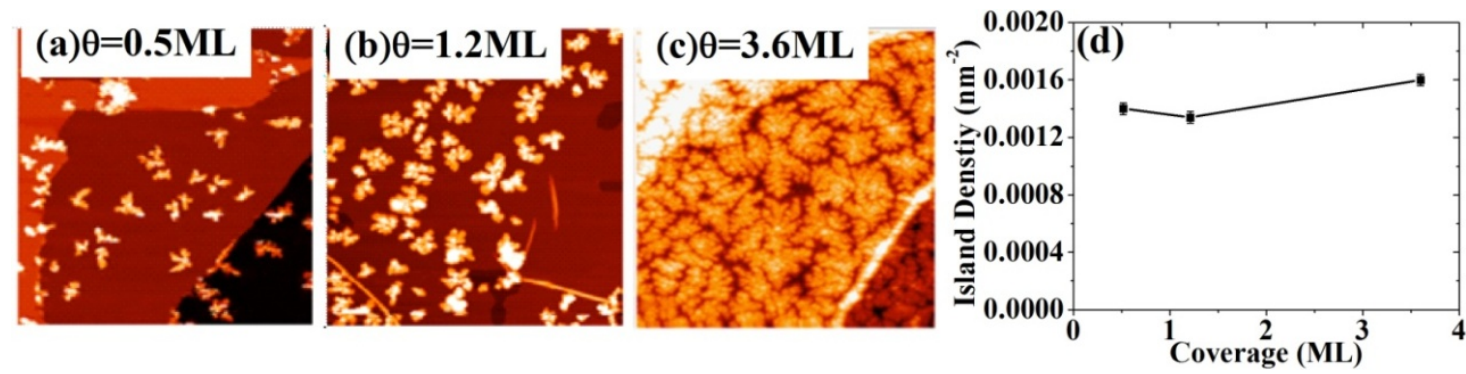

\subsection{Dy on Graphene}

Figure 3a shows the nucleated island density after $0.4 \mathrm{ML}$ of Dy has been deposited at room temperature over an area of $1000 \times 1000 \AA^{2}$.

Figure 3. (a) The nucleated island density after $0.4 \mathrm{ML}$ of Dy has been deposited at room temperature over an area of $1000 \times 1000 \AA^{2}$. The island density is $2 \times 10^{-4}$ islands $/ \AA^{2}$ indicating slow diffusion; (b) Dy $(2.35 \mathrm{ML})$ deposited on graphene with a flux rate $0.23 \mathrm{ML} / \mathrm{min}$ at $660 \mathrm{~K}$. The area size is $2500 \times 2500 \AA^{2}$. These islands have height difference multiples of $0.28 \mathrm{~nm}$, the Dy step height.

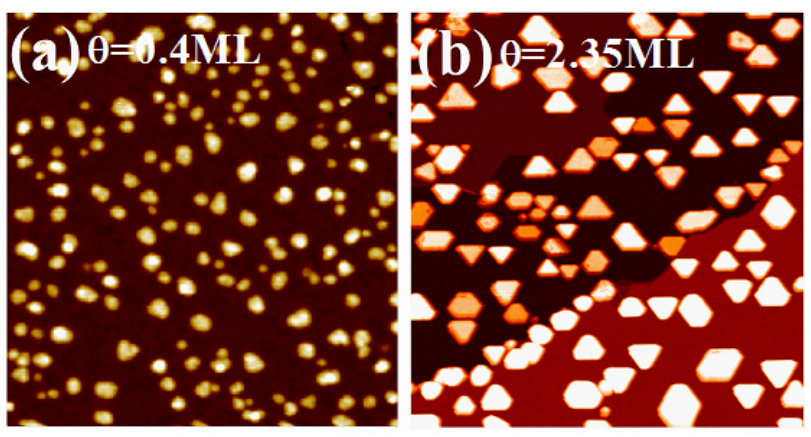


The island density is $2 \times 10^{-4}$ islands $/ \AA^{2}$. However, when Dy is deposited at high temperature of $660 \mathrm{~K}$, bigger and well-shaped islands have been observed as one can see from Figure $3 \mathrm{~b}$ where $2.35 \mathrm{ML}$ of Dy grown with a flux rate of $0.23 \mathrm{ML} / \mathrm{min}$ is shown. The area shown in Figure $3 \mathrm{~b}$ is $2500 \times 2500 \AA^{2}$. These islands are consistent with both hcp(0001) and fcc(111) structure of Dy, and the average step height is $1.93 \mathrm{~nm}$ which corresponds to $7 \mathrm{ML}$ height islands. Most of the islands have triangular shapes which should indicated step free energy anisotropy. For fcc metals, differences in island shapes and orientation are caused either by different stacking sequences or differences in $\mathrm{A}$ and B step surface energies. For hcp(0001) there is only one stacking possibility so the origin of the shape anisotropy is under investigation and most likely it will imply that the Dy nanoislands are fcc(111).

\subsection{Eu on Graphene}

On the other hand, continuous deposition of Eu very quickly results in flat films of large terraces seen in Figure 4 with only two or three layers exposed. Eu, surprisingly, shows flat top crystalline islands with well-defined facets already at RT deposition which can be found in Figure 4. Figure 4a shows $250 \times 250 \mathrm{~nm}^{2} \mathrm{STM}$ images for $\theta=1 \mathrm{ML}$ of Eu deposited on graphene at RT with a deposition rate $0.2 \mathrm{ML} / \mathrm{min}$. The islands are large and crystalline of main height $0.75 \mathrm{~nm}$ with well-defined facets. The film closes in a layer-by-layer fashion if the deposition of Eu continues to $3 \mathrm{ML}$ has been shown in Figure 4.

Figure 4. (a) $250 \times 250 \mathrm{~nm}^{2} \mathrm{STM}$ images for $\theta=1 \mathrm{ML}$ of Eu deposited on graphene at RT with a deposition rate $0.2 \mathrm{ML} / \mathrm{min}$. The islands are large and crystalline of main height $0.75 \mathrm{~nm}$ with well-defined facets; (b) The film closes in a layer-by-layer fashion if the deposition of Eu continues to $3 \mathrm{ML}$ as shown in the STM image of $250 \times 250 \mathrm{~nm}^{2}$.
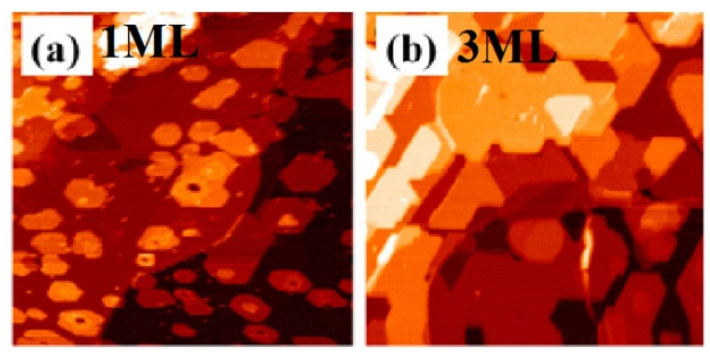

\subsection{Fe on Graphene}

The deviation of Fe nucleation from the standard expectations is seen in Figure 5. For room temperature deposition, $n$ continues to increase even up to 2.3 ML (55\% covered area because the nucleated islands are 3D) and possibly beyond. A continually rising $n$ requires a steady nucleation of new islands, which are seen in the form of small islands in Figure 5a-e. $\theta$ is varied over 3 orders of magnitude from 0.002 to $2.3 \mathrm{ML}$. It is remarkable that for very low $\theta$, a very low $n$ is observed (with these initial Fe islands still $3 \mathrm{D}$ and containing several hundred atoms). This shows that initially the diffusion length is at least $50 \mathrm{~nm}$ (the average island separation in Figure 5a), since the large number of deposited atoms incorporated in the islands must traverse such large distances. However, as more Fe atoms are deposited (see Figure 5b-e) the diffusion length must continuously decrease while $n$ correspondingly increases. 
Figure 5. (a-e) Corresponding images at different $\theta$. (a) $0.003 \mathrm{ML}$; (b) $0.16 \mathrm{ML}$; (c) $0.65 \mathrm{ML}$; (d) $0.87 \mathrm{ML}$; (e) $2.3 \mathrm{ML}$. Small islands in each image show the new islands that have just nucleated. All images are $200 \times 200 \mathrm{~nm}^{2}$ except (b) which is $150 \times 150 \mathrm{~nm}^{2}$; (f) Island density $n$ vs. $\theta$ for Fe grown on graphene at room temperature showing a monotonic dependence on $\theta$ up to at least $55 \%$ surface coverage $(\theta=2.3 \mathrm{ML})$.
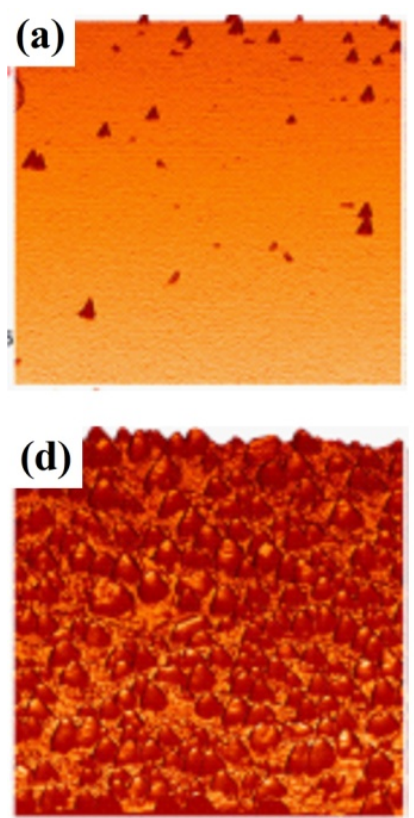
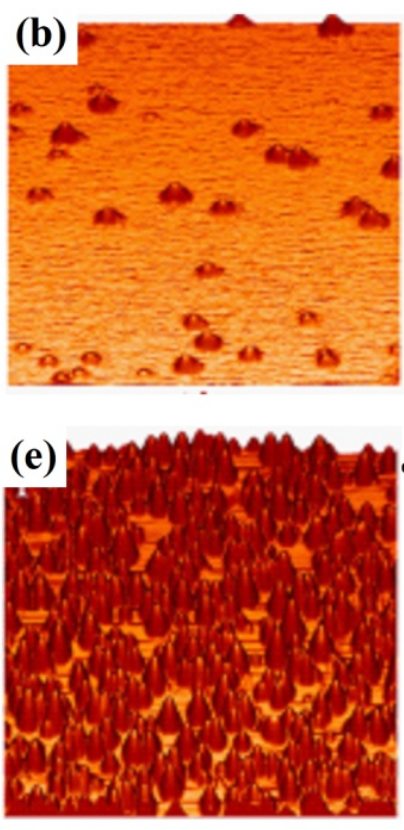

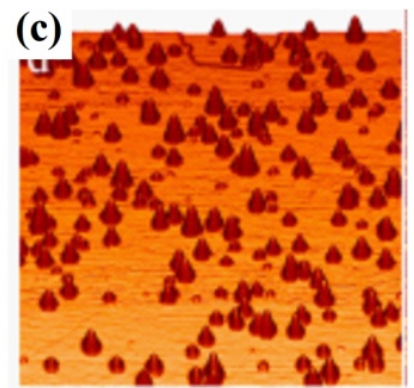

$\%$ of surface coverage

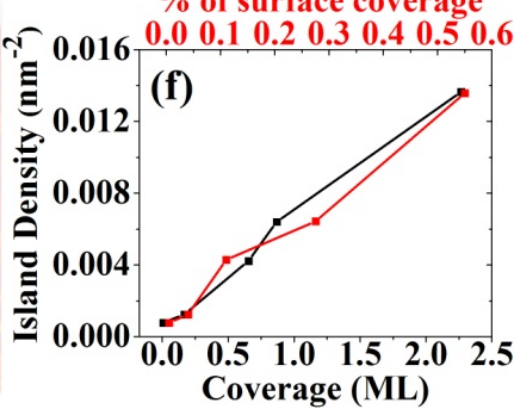

Figure 6 shows the dependence of $n$ on the deposition temperature T from $300 \mathrm{~K}$ to $650 \mathrm{~K}$. Despite the large temperature change $\Delta \mathrm{T}=350 \mathrm{~K}$, the island density is almost constant and fluctuates around its mean value $\sim 6 \times 10^{-4}$ islands $/ \mathrm{nm}^{2}$ by less than a factor of 2 . The temperature dependence of the island density is very weak and is not consistent with activated thermal diffusion. In Figure $5 \mathrm{a}-\mathrm{c} \theta$ is $\sim 0.6 \mathrm{ML}$ and in Figure $5 \mathrm{~d}$ it is $1.05 \mathrm{ML}$. On the other hand, for $\mathrm{Fe} / \mathrm{Fe}(001)$ at $300 \mathrm{~K}$ [102], $n$ is $6 \times 10^{-3}$ islands $/ \mathrm{nm}^{2}$ at RT dropping to $10^{-3}$ islands $/ \mathrm{nm}^{2}$ at $550 \mathrm{~K}$. Nucleation theory [100] was used to extract the diffusion barrier $\Delta E=0.45 \mathrm{eV}$ and normal prefactor $v_{0} \approx 10^{12} \mathrm{hops} / \mathrm{s}$.

Figure 6. The temperatures and converges are (a) RT, $0.65 \mathrm{ML}$; (b) 420K, $0.65 \mathrm{ML}$; (c) $570 \mathrm{~K}, 0.54 \mathrm{ML}$; (d) $650 \mathrm{~K}, 1.05 \mathrm{ML}$; (e) Temperature dependence of the island density $n$ from 300 to $650 \mathrm{~K}$. Classical nucleation predicts an Arrhenius dependence of $n$ with temperature while images (a-d) instead show an almost constant island density. Each image is $200 \times 200 \mathrm{~nm}^{2}$.
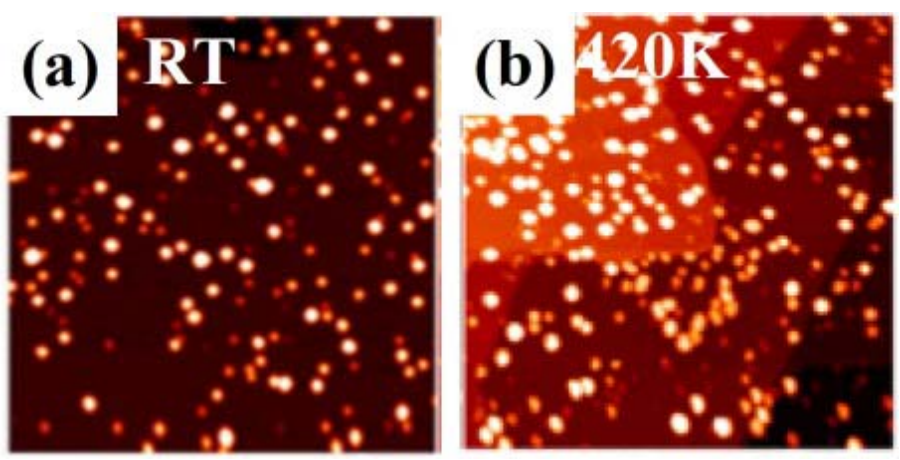
Figure 6. Cont.

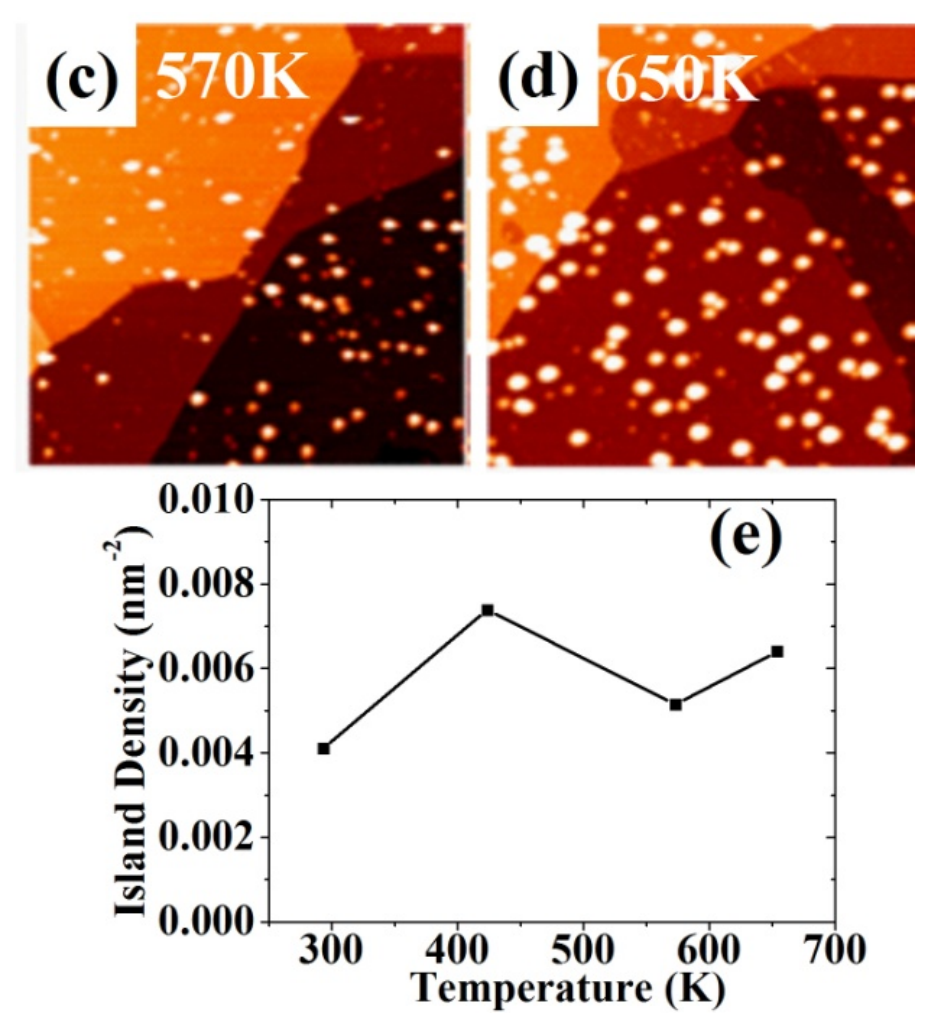

\subsection{Pb on Graphene}

Figure $7 \mathrm{a}$ shows a $1000 \times 1000 \AA^{2}$ area with only two large $\mathrm{Pb}$ islands with growth conditions $\mathrm{T}=78 \mathrm{~K}$ and $\theta=0.5 \mathrm{ML}$. The islands have heights of eight layers and regular face-centered cubic (fcc) (111) shapes. It is clear that $\mathrm{Pb}$ diffusion is extraordinarily fast since all the deposited atoms have moved to the islands. Using an island density of $2 \times 10^{-6}$ islands $/ \AA^{2}$, a flux rate of $0.1 \mathrm{ML} / \mathrm{min}$, the scaling theory of nucleation [100] with critical size clusters $i_{\mathrm{c}}=1$, and a normal prefactor $10^{13} \mathrm{~s}^{-1}$, it is deduced that the diffusion barrier $\Delta E$ much be less than $70 \mathrm{meV}$. This estimated $\Delta E$ is an upper bound because some islands nucleate at steps so the diffusion length is limited by the average distance between steps. This is confirmed with nucleation experiments at a lower temperature $(30 \mathrm{~K})$ that give island densities only slightly higher $\left(4 \times 10^{-6}\right.$ islands $\left./ \AA^{2}\right)$ than the experiments at $78 \mathrm{~K}$. Using this island density the activation energy must be less than $35 \mathrm{meV}$ (but the island shapes grown at $30 \mathrm{~K}$ are less regular than the ones in Figure 7a).

The absence of monomers on top of graphene is seen in Figure $7 b$ where an area of $540 \times 440 \AA^{2}$ around the top island of Figure $7 \mathrm{a}$ is magnified. The periodic pattern observed around the island corresponds to the $18 \AA$ periodicity of the $6 \sqrt{3}$. Since there is no wetting layer, no monolayer rings are present at island perimeters as for the growth of $\mathrm{Pb} / \mathrm{Si}(111)$ [103]. 
Figure 7. (a) $250 \times 250 \mathrm{~nm}^{2}$ area with only 3 large $\mathrm{Pb}$ islands the temperature is $\mathrm{T}=40 \mathrm{~K}$ flux rate $0.1 \mathrm{ML} / \mathrm{min}$ and deposited amount $\theta=0.8 \mathrm{ML}$. The islands have heights 8-, 6-, 7-layers from left to right. The measured island density $3 \times 10^{-5}$ islands $/ \mathrm{nm}^{2}$ implying a $\mathrm{Pb}$ diffusion barrier less than $50 \mathrm{meV}$; (b) The area is $54 \times 44 \mathrm{~nm}^{2} \mathrm{~Pb}$ after growth at $\mathrm{T}=88 \mathrm{~K}$ and $\theta=0.5 \mathrm{ML}$. The periodic pattern around the 7-layer island is the initial $6 \sqrt{3}$ reconstruction which is practically free of monomers indicating unusually high $\mathrm{Pb}$ mobility.
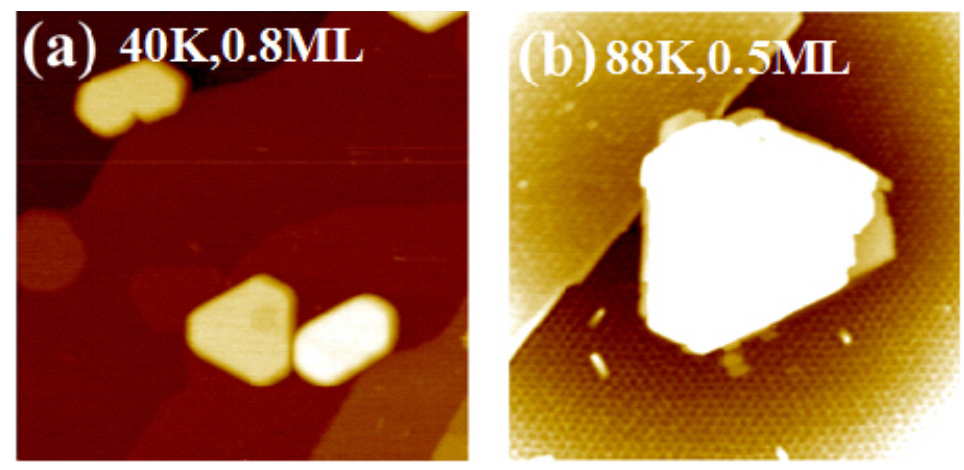

\section{Interactions between Metal Adatoms and Graphene}

As discussed in last section, different metals on graphene exhibit different growth morphologies which can be attributed to the different bonding and interaction strength between the metal and the graphene. Studies of metal adatom adsorption on graphene will provide useful information of these interactions. In the past several years, extensive first-principles calculations based on density functional theory have also been carried out to study the adsorption properties of various metals on graphene in order to gain detailed insights into the interaction of the metals with graphene [31-64]. The first-principles DFT plane-wave basis calculations have also been combined with quasi-atomic minimal basis orbitals (QUAMBOs) scheme [104-106] and Mulliken charge analysis to evaluate the amount of electron transfer between the adatoms and the graphene layer [71-78]. The degree of covalent bonding is also studied through Mayer bond order [107] analysis based on the QUAMBOs. The results from the first-principles calculations and the QUAMBO analysis have provided useful information for a better understanding of the growth behavior of metal nanostructures formation on graphene. The adatom/graphene system is modeled by having one metal adatom in a $6 \times 6$ graphene supercell and with periodic boundary conditions.

\subsection{Energeitcs of Metals on Graphene}

The adsorption energies of various metal adatoms on graphene have been studied by first-principles calculations [31-64]. The calculation results from Liu et al. [71-73,76] show that the energetically favorable adsorption site is different for different adatoms. Most of the metal adatoms favor the $H$-site, while $\mathrm{Pb}, \mathrm{Cu}, \mathrm{Au}$ adatoms prefer the $T$-site and $\mathrm{Cr}, \mathrm{Pd}, \mathrm{Pt}$, and $\mathrm{Ag}$ adatoms take the $B$-site. The adsorption energies $\left(E_{\mathrm{a}}\right)$ and diffusion barrier $(\Delta E)$ for various metal adatom adsorbed on graphene obtained from DFT calculations of Liu et al. are shown in Figure 8a,b, respectively, in comparison with other works $[29,31,38,40,45,48-51,55]$. The metals in the horizontal axis are arranged in the order of group I-IV 
metals, 3d-transition metals, group 10 transition metals, noble metals, as well as rare earth metals. The adsorption energy $E_{\mathrm{a}}$ is defined as the difference between the energy of the relaxed adatom/graphene system and that of the isolated perfect graphene sheet and an isolated adatom. From Figure 8a one can see that the adsorption energy ranges from less than 0.04 to $1.95 \mathrm{eV}$, depending on the type of adatoms. For example, the adsorption energy is much smaller when $\mathrm{Ag}$ and $\mathrm{Mg}$ adsorbed on graphene, only about 0.02-0.03 eV, suggesting that the interactions between $\mathrm{Mg}$ and $\mathrm{Ag}$ adatoms and graphene are very weak. The adsorption of $\mathrm{Mg}$ and $\mathrm{Ag}$ adatoms on graphene is therefore physisiorption. In contrast, the interactions between most of the 3d-transtion metals and graphene are much stronger with adsorption energy ranging from 0.87 to $1.95 \mathrm{eV}$ except for $\mathrm{Cr}$ and $\mathrm{Mn}$ on graphene which has adsorption energies of only 0.21 and $0.14 \mathrm{eV}$, respectively. The adsorption energy is 1.15 and $1.70 \mathrm{eV}$, respectively, for $\mathrm{Pd}$ and $\mathrm{Pt}$ adatoms on graphene. The adsorption energy of group I-IV metals (except $\mathrm{Mg}$ ) on graphene is intermediate from about $0.26-1.17 \mathrm{eV}$. When noble metal adatoms adsorbed on graphene, the adsorption energy is much smaller. In the case of rare earth metals on graphene, the adsorption energy is also generally high (above $1.56 \mathrm{eV}$ ) except for the half-filled and fully-filled f-shell elements $\mathrm{Eu}$ and $\mathrm{Yb}$ which have adsorption energies of 0.87 and $0.35 \mathrm{eV}$, respectively. The results from the first-principle calculations of Liu et al. agree in general with other theoretical results $[29,31,38,40,45,48-51,55]$ as one can see from Figure 8 . We note that, for the alkali metal adsorption, the adsorption energies are smaller in $4 \times 4$ supercell than that of $6 \times 6$ supercell due to Coulomb repulsion effects.

Figure 8. (a) Adsorption energy $\left(E_{\mathrm{a}}\right)$; (b) Diffusion barrier $(\Delta E)$. The bars correspond to the work by the current calculations and the symbols to results in the literature.

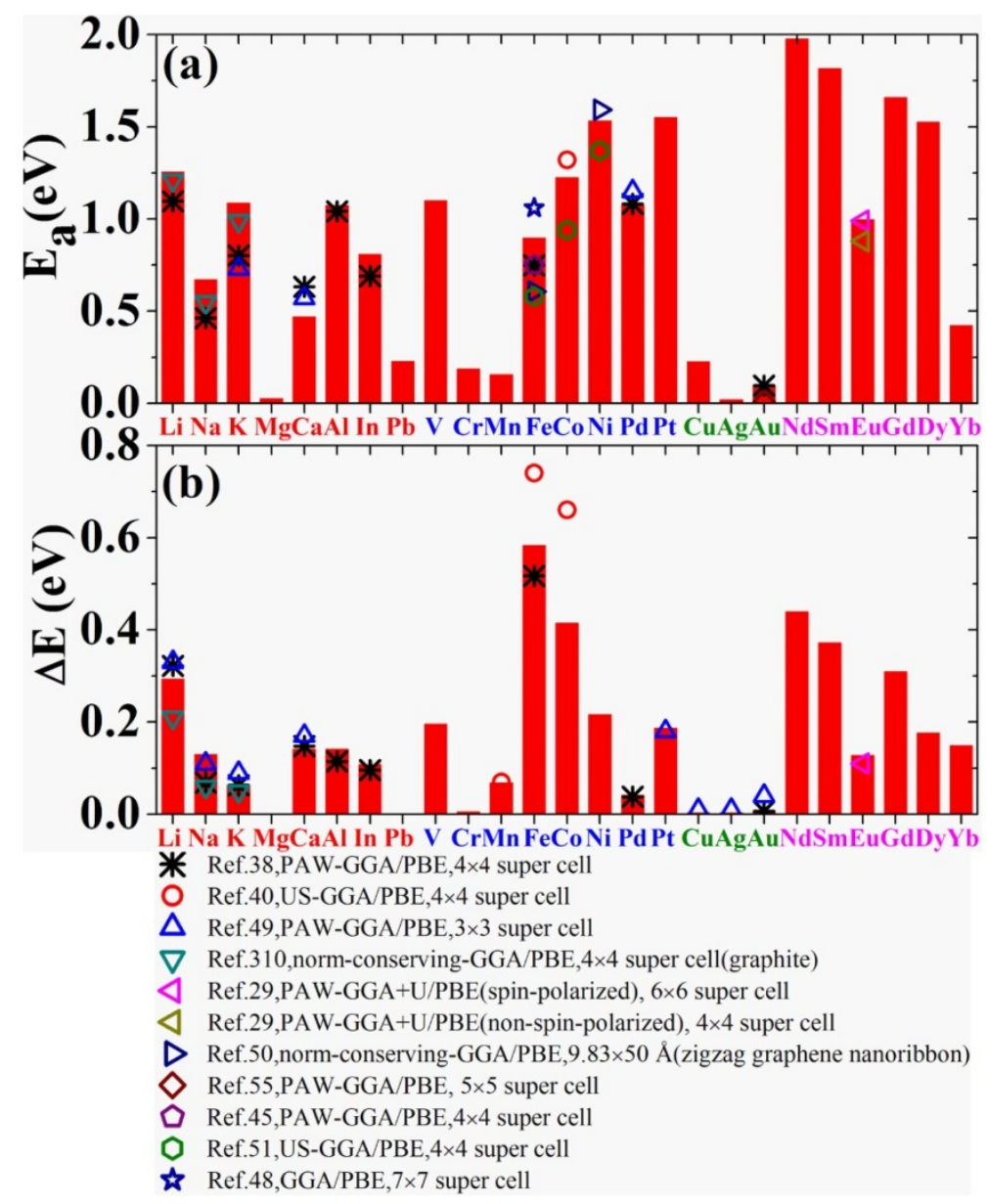


Figure $8 \mathrm{~b}$ shows the diffusion barriers for different metal adatoms on graphene obtained from the first-principles calculations of Liu et al., also in comparison with other calculation results $[38,49,71,76]$. The diffusion barriers are very small with only few meV for $\mathrm{Mg}, \mathrm{Pb}, \mathrm{Cr}, \mathrm{Cu}, \mathrm{Ag}$, and $\mathrm{Au}$ adatoms on graphene. For group I-IV metals, the diffusion barriers are generally lower with the values from 0.06 to $0.14 \mathrm{eV}$, except for a $\mathrm{Li}$ adatom which has a diffusion barrier of $0.32 \mathrm{eV}$. On the other hand, the diffusion barriers for 3d-trnasitoin metal adatoms on graphene are generally higher [71], except for $\mathrm{Cr}$ and $\mathrm{Mn}$ adatoms. The diffusion barriers of $\mathrm{Cr}$ and $\mathrm{Mn}$ adatoms on graphene are 0.01 and $0.03 \mathrm{eV}$ respectively [76]. Compared to the $3 \mathrm{~d}$-transiton metals on graphene, the diffusion barriers of noble metals on graphene are much smaller, only few meV [76]. For rare earth metals, the diffusion barrier of $\mathrm{Nd}, \mathrm{Sm}$, and Gd adatoms are generally higher compared to that of Eu, Dy, and $\mathrm{Yb}$ adatoms $[72,76]$ as one can see from Figure $8 \mathrm{~b}$.

\subsection{Interaction Charge Density Distribution}

The adsorption energies and diffusion barriers can be used as a measure for the interaction between metal adatom on graphene: small adsorption energy and diffusion barriers indicated weak interaction and vice versa. The nature (i.e., ionic or covalent) and the strength of the interaction can be qualitatively understood by the analysis of bonding electron distributions (BED) as shown in Figures 9 and 10. Here the BED $\Delta \rho(r)$ is defined as $\Delta \rho(r)=\rho(r)-\left(\rho_{\text {gra }}(r)+\rho_{\text {ada }}(r)\right)[8,46,76]$, where $\rho(r)$ is the electron density of the adatom/graphene system, $\rho_{\text {gra }}(r)$ and $\rho_{\text {ada }}(r)$ are the electron density of the pure graphene and the isolated adatoms separately calculated using the same atomic coordinates and supercell setup as those in the corresponding adatom/graphene systems. The BED accounts for the charge redistribution due to the interaction between the adatom and graphene. A three-dimensional (3D) plot of the BED for various metal adsorptions is shown in Figure 9.

Figure 9. Bonding electron distribution (BED) induced by adatoms adsorption on graphene. The gold color (i.e., $0.004 \mathrm{e} / \AA^{3}$ ) in the 3D plot indicates and increase in the electron density after bonding, and the cyan color (i.e., $-0.004 \mathrm{e} / \AA^{3}$ ) indicates and electron density loss.

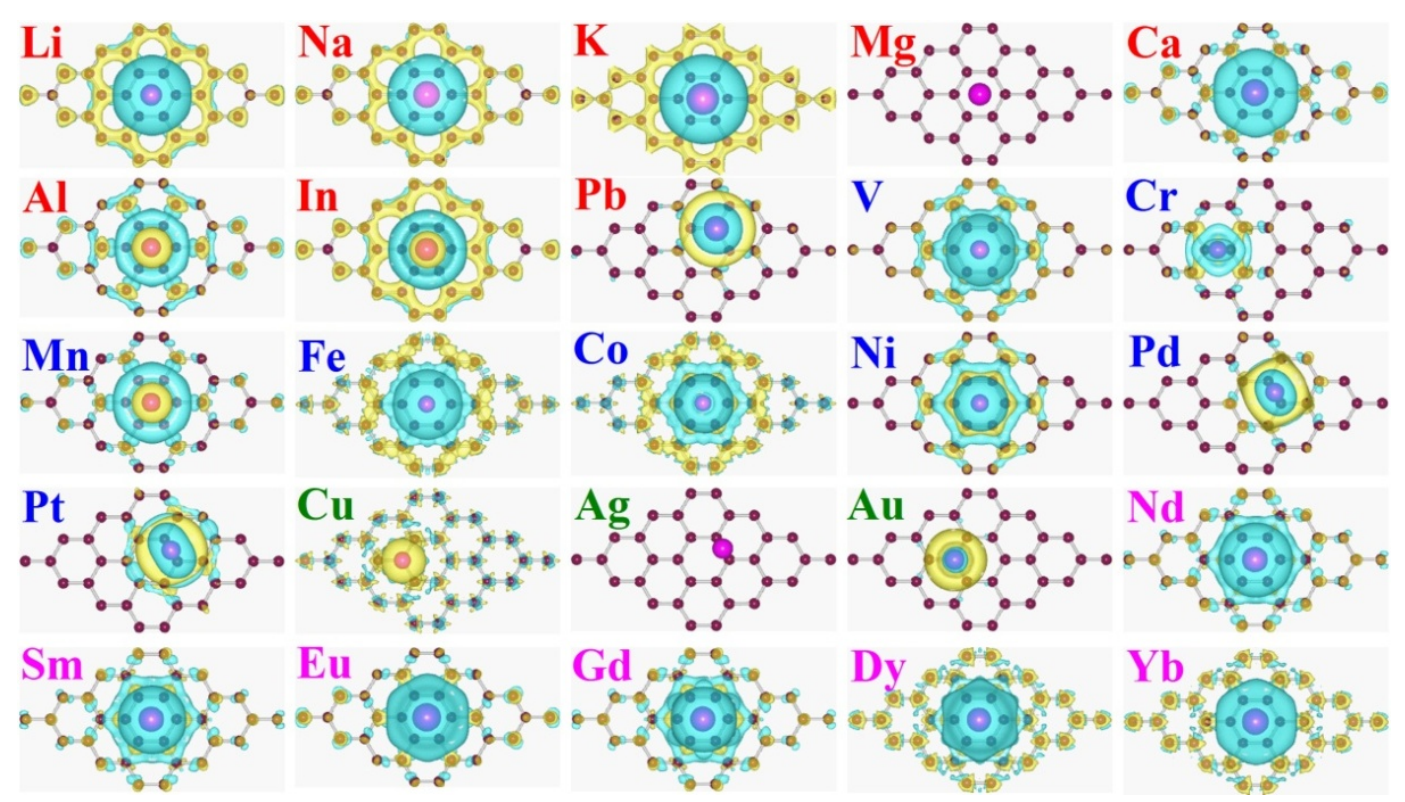


The gold color (i.e., $0.004 \mathrm{e} / \AA^{3}$ ) in the $3 \mathrm{D}$ plot indicated an increase in the electron density upon adsorption, and the cyan color (i.e., $-0.004 \mathrm{e} / \AA^{3}$ ) indicated an electron density loss. A two-dimensional (2D) contour plot of the BED in the vertical planes cutting through the adatom and the carbon atoms of the graphene layer is also shown in Figure 10. The region with a higher bonding charge between adatoms and carbon atoms in Figures 9 and 10 indicates stronger covalent bond formation by adatom adsorptions. As shown in Figure 9, the bonding charge distribution induced by simple metal adsorption is delocalized, especially for alkali metal adatoms. The electrons transferred from alkali metal adatoms spread over the whole graphene layer. In comparison, the induced bonding electron density by 3d-transiton, group 10 transition metals, and noble metals on graphene is mainly localized on the carbon atoms next to the metal adatoms. For the rare earth metal adatoms, the bonding charge distribution (expect for $\mathrm{Yb}$ ) is intermediate between the alkali and 3d-transition metal, i.e., the $\Delta \rho(\mathrm{r})$ exhibits some degree of localization but is not as strong as those of transition metals on graphene. For $\mathrm{Yb}$ on graphene, the bonding charge distribution is delocalized due to charge transfer since the interaction between the $\mathrm{Yb}$ adatom and graphene is ionic. The bonding charge distribution is similar to the case of alkali metals on graphene and spreads over the whole graphene layer.

Figure 10. Bonding electron distributions (BED) in the vertical plane cutting through adatom and carbon atoms of the adatom/graphene system. Positive value (red color indicated 0.28 electron $/ \AA^{3}$ ) indicates an increase in electron density and negative value (blue color indicated -0.14 electron $/ \AA^{3}$ ) means an electron density loss with respect to the superposition of the atomic electron density.

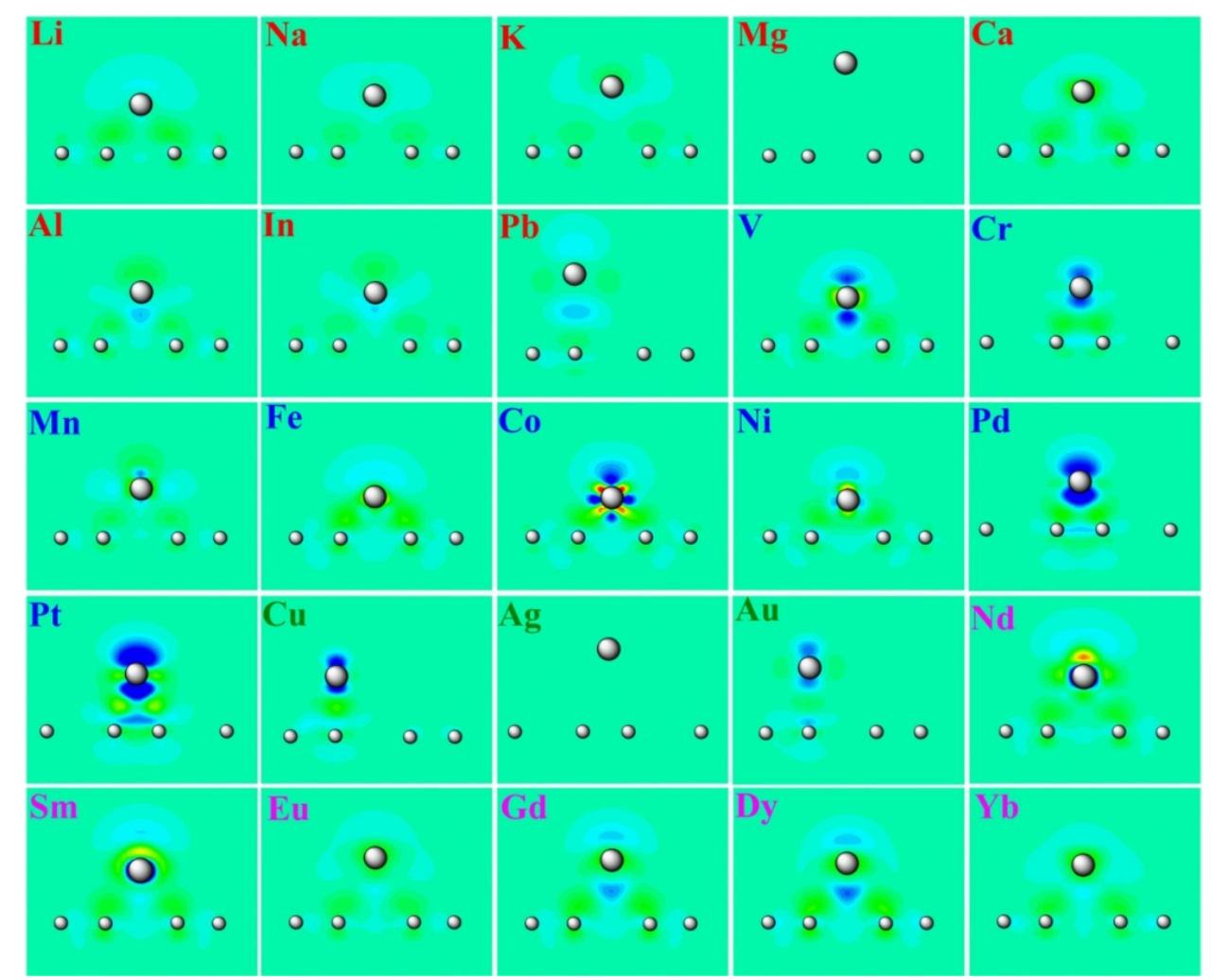




\subsection{Microscopy Bonding Nature and Mechanism}

More quantitative charge transfer and microscopic bonding mechanism for metals on graphene have also been analyzed through the popular Mulliken population analysis by expressing the wave functions from the first-principles calculations in terms of QUAMBOs by Liu et al. [71-73,76]. Figure 11a presents the charge transfer induced by different metal adatoms on graphene. We can see that for alkali metal adatom on graphene, almost all their $s$ electron is transferred to graphene. Chan et al. [38] also analyzed the electron transfer between the alkali and simple metal adatoms and graphene using two different methods: (i) They determine the charge transfer by integrating the electron density in the adatom region and graphene region respectively within the supercell based on the results from the DFT calculations using the plane-wave basis; and (ii) They determine the charge transfer from the electronic density-of-state (DOS) according to the difference between the Fermi level in pure graphene and metal adsorbed graphene systems. They [38] showed that the charge transfer is only about $0.4 \sim 0.7$ electrons for alkali adatoms on graphene, which is lower than the value of 1.0 electron found by the calculation of Liu et al. The smaller charge transfer from their calculations can be attributed to the size effects since Coulomb repulsive interactions between adatoms and their images in the $4 \times 4$ super cell can reduce the electron transfer. The work of Liu et al. [71,76] and the recent studies [12-14,31,38] showed that alkali adatoms adsorption on graphene donate their s electron easily and act as good agents for $n$-doping of graphene. Therefore the interaction between alkali adatoms and graphene is mainly ionic. For the $\mathrm{Mg}$ /graphene system, Liu et al also found that [76] there is almost no charge transfer, consistent with the physisorption picture discussed above. By contrast, they found there are about 1.33 electrons transferred from $\mathrm{Ca}$ to graphene. For group III and IV metals, about 0.8-0.9 electron transfers are found for $\mathrm{Al}$ and In adatoms on graphene while the charge transfer is only 0.3 electrons of the $\mathrm{Pb}$ adatom from the studies $[38,83,86]$. For $3 \mathrm{~d}$-transtion metals on graphene, the charge transfer is found below 1.0 electron, except for the Fe/graphene system in which transfer of 1.2 electrons has been found. Larger charge transfer for Fe/graphene is also reported in [40]. The charge transfer by adsorption of noble metals is relatively smaller with the values no more than 0.4 electrons. Similar results are also found in other works [54-56]. In the case of rare earth metals, the charge transfer depends on the adatom type. The charge transfer is 1.34 electrons for $\mathrm{Yb}$, but relatively smaller for other rare earth metals on graphene [72,76].

The nature of the bonding for metal on graphene has also been discussed in term of bond order [102]. The bond orders are calculated through the QUAMBOs method by Liu et al. [71-73,76] and the results are shown in Figure 11b. One can see that more than a half of the metals in the region right have a bond order value of about 1.5 , indicating strong covalent bond formation between these adatoms and graphene [71-73,76]. Those adatoms have strong covalent interaction with graphene also have relatively large adsorption energy and diffusion barriers. Another half of metal adatoms on the right side of Figure $11 \mathrm{~b}$ exhibit smaller bond orders (less than 0.75) as well as smaller adsorption energies and diffusion barriers. Ag adatom is exceptional for which the bond order and adsorption energy are all zero, indicating that there is no noticeable interaction between the $\mathrm{Ag}$ adatom and graphene. They $[71-73,76]$ also note that the bond orders of the alkali and group II metal adatoms are essentially zero, indicating ionic bonding between these adatoms and graphene. In particular, $\mathrm{Mg}$ adatom also exhibit negligible adsorption energy and diffusion barrier, showing physisorption 
characters. In comparison, the bond orders of the groups III and IV metal adatoms (Al, In, and $\mathrm{Pb})$ are moderate with a mix of ionic and covalent interactions with graphene. They also note that the adsorption of transition metal adatoms on graphene cause strong hybridization between the transition metal adatoms and graphene electronic states and the bonding is mainly covalent $[71-73,76]$.

Charge transfer upon metal adsorption on graphene has also been investigated by experiments [10-14,31-37]. Recent experimental studies showed that alkali adatoms adsorption on graphene donate their valence electron easily (i.e., 0.3 0.4 electrons) and act as good agents for $n$-doping of graphene [10-14,31-37]. By using the STM technique combined with density functional calculations, Song et al. [10] studied cesium adsorption on graphene supported on a $6 \mathrm{H}-\mathrm{SiC}(0001)$ substrate. They showed that the interaction between $\mathrm{Cs}$ adatoms on graphene originates from a long-range repulsive Coulomb interaction due to the charge transfer from Cs to graphene. Such an electrostatic potential couples with the substrate potential to form stable Cs superlattices. Virojanadara et al. [11] also found by ARPES experiment studies that Li adatom give rise to electron doping of graphene but in addition they showed that the Dirac cone is sensitive to how the Li atoms are distributed on the graphene $\mathrm{SiC}$ interface after room temperature intercalation. The interaction between $\mathrm{K}$ adatoms on graphite also exhibited long range repulsive interactions from a STM study [14] but the coverage was very low and the temperature much lower so no ordered arrangements of the $\mathrm{K}$ adatoms were accessible. All the experiments [10,11,14] and the theoretical studies [31-37] showed that alkali adatoms adsorption on graphene donate their electrons easily. Therefore the interaction between alkali adatoms and graphene is mainly ionic, which is consistent with the conclusions of Liu et al. $[71-73,76]$ and other works [12,13,31,38].

Figure 11. (a) Charge transfer $(\Delta q)$ due to adatom adsorption; (b) Bond order of adatom/graphene system.

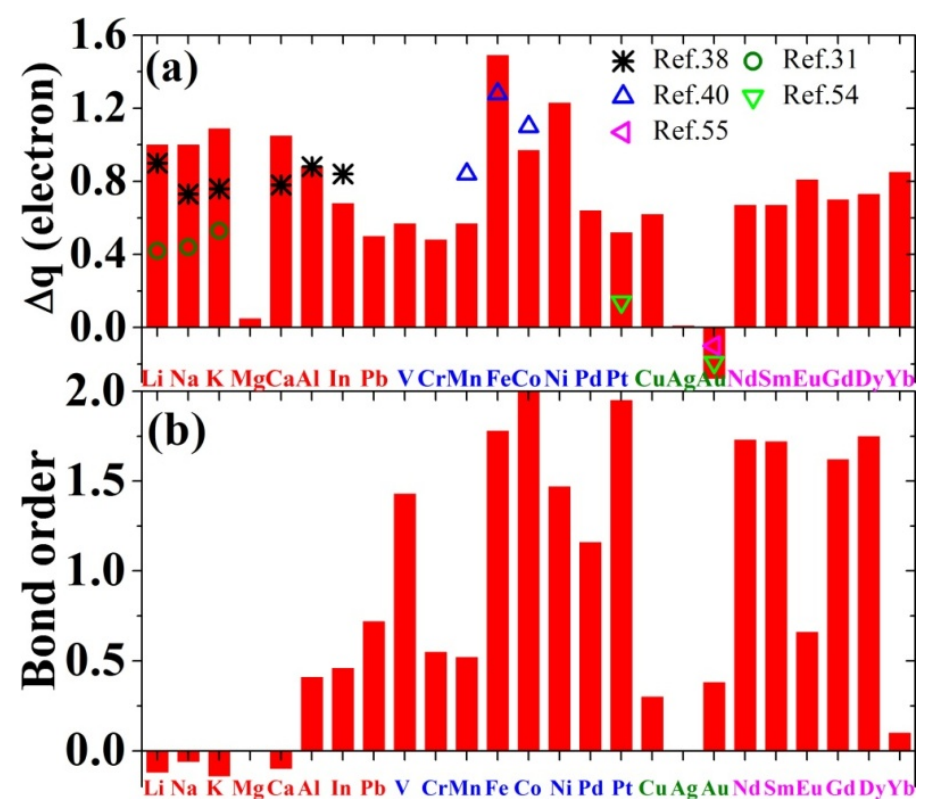

\section{Properties Induced by Metal Atom Adsorption}

Metal adsorption can induce changes in the structures and properties of graphene which in turn can affect the growth morphology of metal on graphene. Therefore, before discussing the correlation 
between the growth morphology and adatom adsorption properties in Section 5, we will review some current investigations on adatom induced properties for metal on graphene.

\subsection{Deformation of Graphene}

An important property induced by the metal adatom adsorption on graphene is the lattice distortion. Such lattice distortions would cause elastic interactions between the adatoms and affect the growth morphologies of the metal on graphene. Different metal adatoms on graphene can cause different distortions. In Figure 12, the in-plane distortions of the graphene layer upon the adsorption of the metal adatoms from the calculation of Liu et al. $[71-73,76]$ are plotted. In order to see the distortions more clearly, the displacements indicated by the blue arrows in Figure 12 have been enlarged by a factor of 5000 for a better visualization. Liu et al. $[71-73,76]$ found that the distortion patterns (or strain fields) in the graphene layer caused by the adsorption of group I-IV metals are very similar. The lattice distortion of graphene caused by the adsorption of 3d-transition and group 10 transition metals is larger compared to that induced by group I-IV metal adsorptions as one can see from Figure 12. For noble metal adsorptions, the distortion induced by $\mathrm{Cu}$ is much larger than that by $\mathrm{Ag}$ and $\mathrm{Au}$ adatoms on graphene. For rare earth metals on graphene, there is very smaller in-plane distortion induced by adsorption of $\mathrm{Eu}$ and $\mathrm{Yb}$. The in-plane distortion induced by other rare earth metals is also not as large as that by 3d-transiton metals but larger than that by group I-IV metals. They note that the in-plane distortion of the second shell carbon atoms with respect to the adatoms is larger than that of the first shell carbon atoms. These distortion patterns (or strain fields) induced by the adatom adsorption would affect the growth morphology of metals on graphene.

Figure 12. In-pane distortion of graphene lattice due to the adsorption of metal adatoms. The length of the arrows represents the amplitude of the distortion and the direction of the arrows represents the direction of the distortion. The displacements have been enlarged by a factor of 5000 for a clear visualization.

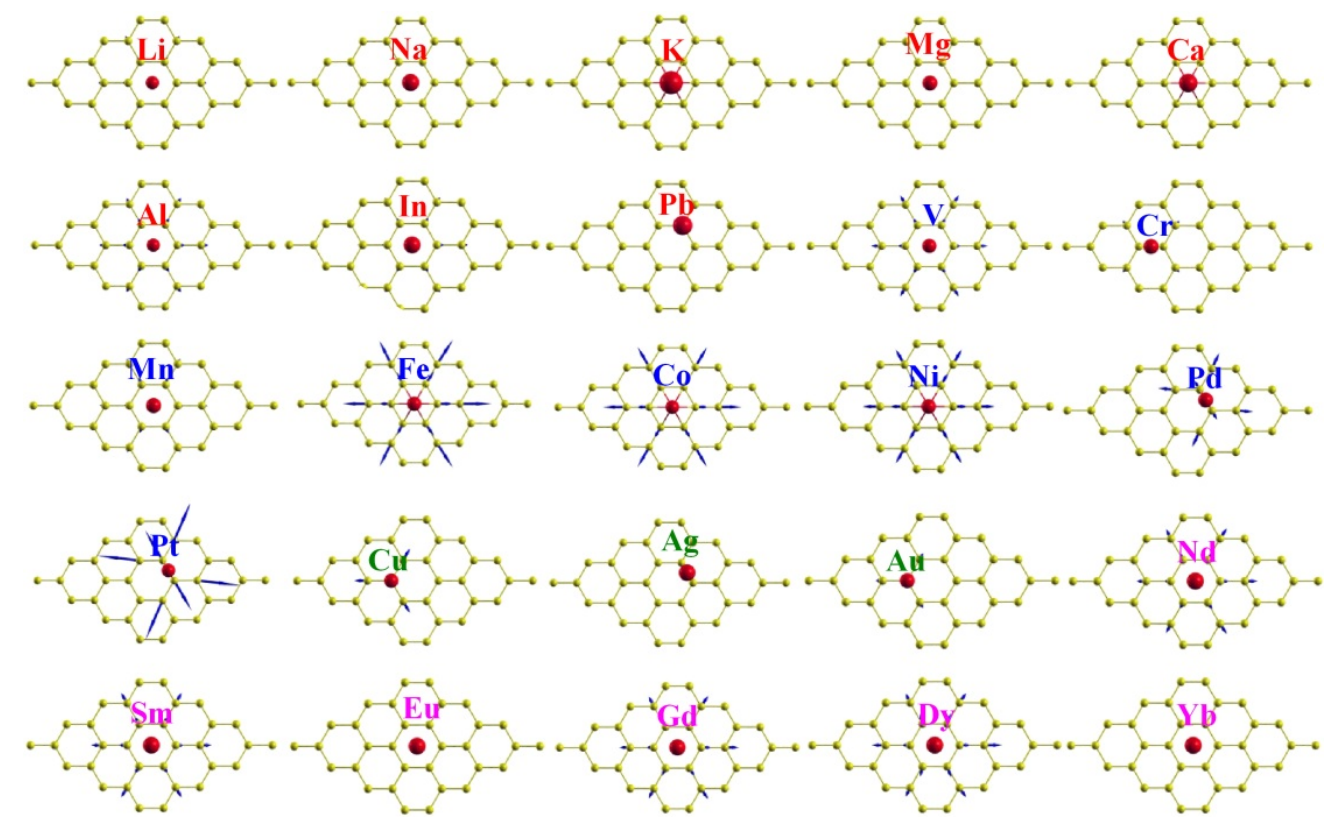




\subsection{Electric Dipole Moment}

The redistribution of electrons by adsorption of metals on graphene will induce electric dipole moments, which cause long-range interaction between the adatoms on the graphene and affect the metal island growth morphology. The electric dipole moments from the calculations of Liu et al. [71-73,76] are summarized in Figure 13a. They show that $[71-73,76]$ the dipole moment induced by alkali metals is larger with the values ranging from 2.5 to 6.0 Debye due to charge transfer which is consistent with the results of Chan et al. suggested [38]. But for group II-IV metals on graphene, the dipole moment is below 1.0 Debye except for In adsorption on graphene which gives 2.5 Debye of dipole moment. The dipole moment is about 1.0 Debye when $\mathrm{V}, \mathrm{Fe}, \mathrm{Co}$, and Ni adatoms deposit on graphene. However, the other 3d-transiton metals, for example, $\mathrm{Cr}$ and $\mathrm{Mn}$ adatoms, induce little dipole moment with the values only $0.1-0.2$ Debye. The induced dipole moment by adsorption of $\mathrm{Pd}$ and $\mathrm{Au}$ is about 1.2 Debye, respectively [76]. In the case of $\mathrm{Pt}, \mathrm{Cu}$, and $\mathrm{Ag}$ adsorption, the dipole moment is smaller. The dipole moment of rare earth metals on graphene is relatively larger, especially for $\mathrm{Nd}, \mathrm{Gd}$, and $\mathrm{Dy}$ adsorption with the values of 2.6, 1.9, and 1.8 Debye, respectively [72,76]. Some correlations between the amount of the charge transfer and electric dipole moment can be seen from the comparison of Figures 13a and 12a. In general, the larger the charge transferred, the bigger the induced electric dipole moment but with several exceptions. For example, $\mathrm{Ca}, \mathrm{Al}$, and $\mathrm{Yb}$ adatoms have relatively larger charge transfer, but their electric dipole moments are small.

Figure 13. (a) Electric dipole moments; (b) Magnetic moment for metal/graphene system (blue bar) and isolated metal atoms (red bar).

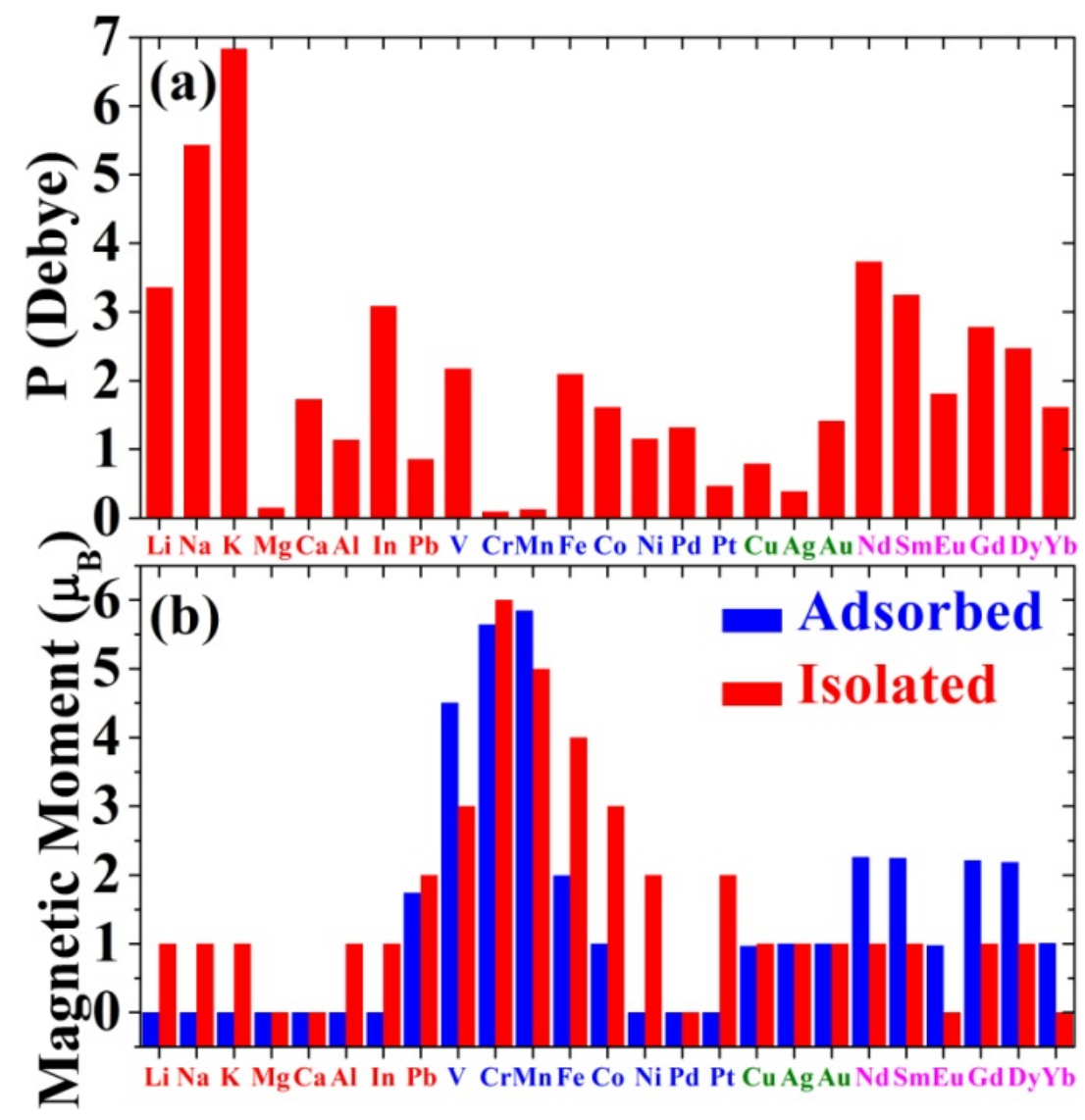




\subsection{Magnetic Moment}

The charge transfer between metal adatoms and graphene as well as among the different orbitals of the adatoms may also induce spin-polarization and thus magnetic moment. Figure 13b shows the magnetic moments of metal/graphene system in comparison with those of corresponding isolated metal atoms from the studies of Liu et al. [71-73,76]. For Li, $\mathrm{Na}$ and $\mathrm{K}$ adatoms on graphene, the magnetic moments are disappeared, showing a significant reduction from their values of $1.0 \mu_{\mathrm{B}}$ in the isolated atoms. The origin of this magnetic moment reduction can be attributed to the electron transfer from the s orbital of the alkali metal adatoms to graphene suggested by Liu et al. [71,76]. There is one $s$ electron in an isolated alkali metal atom which gives $1.0 \mu_{\mathrm{B}}$ of the magnetic moment. However, when the alkali metal atom adsorbs on graphene, almost its valence electron is transferred to the $p_{z}$ orbitals of the graphene and reduce the magnetic moment of the s electron thus no net magnetic moment for alkali metals on graphene. The isolated $\mathrm{Mg}$ and $\mathrm{Ca}$ metal atoms exhibit zero magnetic moment due to closed shell of their electron configuration. However, there is no charge transfer between $\mathrm{Mg}$ and graphene layer so the net magnetic moment of $\mathrm{Mg}$ /graphene system is zero, as one can see from Figure 13b. For $\mathrm{Pb}$, the adsorption slightly reduces the magnetic moment from its atomic value of $2.0 \mu_{\mathrm{B}}$ to $1.77 \mu_{\mathrm{B}}[73,76]$. There are no net magnetic moments for $\mathrm{Al}$ and $\mathrm{In}$ adatoms on graphene as suggested by Liu et al. and Chan et al. $[38,71,76]$. In comparison, the odd number of $p$ electrons in the isolated atoms will give $1.0 \mu_{\mathrm{B}}$ of magnetic moments. The depletion of magnetic moments can also be attributed to these electron transfers. Depletion of magnetic moments is also observed for the 3d-transtion metal adatoms (except V and Mn) on graphene [38,40,48,51,71,76]. Recently, a STM investigation [26-28] revealed that the interface magnetic properties of nanostructured transition metal $\mathrm{Co} /$ graphite can be used to determine the spin and orbital moments at the hybrid interface. Vo-van et al. [26,27] reported the growth of flat, epitaxial ultrathin transition metal Co films on graphene on $\operatorname{Ir}(111)$ substrate for a candidate graphene-based materials in spintronics.

For the noble metal adatoms on graphene, the net magnetic moment of the system is equal to the value of those isolated noble metal adatoms [76] as one can see from Figure 13b. It is worthy to note that the magnetic moment of the rare earth metal adatoms on graphene is significantly enhanced [72,76]. Liu noted that the core f-electrons of the rare earth adatoms are not included in the VASP calculations as discussed in [72]. Therefore the magnetic moments presented in Figure 13b do not include those from the core f-electrons. They show that the magnetic moments of the rare earth adatoms are generally higher than those of the isolated atoms. For $\mathrm{Nd}, \mathrm{Sm}, \mathrm{Gd}$, and Dy while the magnetic moments from the valence electrons of the isolated atoms are about $1.0 \mu_{\mathrm{B}}$, the adatom/graphene system exhibits a magnetic moment of $2.0 \mu_{\mathrm{B}}[72,76]$. Similarly, the adsorption of Eu and $\mathrm{Yb}$ on graphene also induces a magnetic moment of $1.0 \mu_{\mathrm{B}}$ although there is no magnetic moment from the valence electrons in the isolated $\mathrm{Eu}$ and $\mathrm{Yb}$ atoms [72,76]. The enhancement of magnetic moment upon adsorption of rare earth adatoms on graphene is in contrast to the behaviors of most of the simple and transition metals where the magnetic moments of the adatoms are reduced upon adsorption on graphene. Their calculations also show that magnetic moments of the graphene layer are enhanced significantly by the adsorption of the rare earth adatom, suggesting that the magnetic properties of graphene can be manipulated through the doping or adsorption. 


\section{Correlation between Growth Morphology and Adatom Adsorption Properties}

In order to understand the growth morphology of the metal nanostructures on graphene, Liu et al. [71-78] have examined the adsorption energy of the metal adatom on graphene with respect to its bulk cohesive energy $\left(E_{\mathrm{a}} / E_{\mathrm{c}}\right)$ as well as diffusion barriers $(\Delta E)$ of adatom on graphene. They showed that there are correlations between growth morphology and $E_{\mathrm{a}} / E_{\mathrm{c}}$ as well as $\Delta E$.

Although the details of the growth morphology would be dependent on many energetic and kinetic factors, it is plausible to argue that the dimensionality aspect of the metal island growth (i.e., 2D or 3D growth) is mainly governed by the ratio of the adsorption energy to the bulk cohesive energy $\left(\mathrm{E}_{\mathrm{a}} / \mathrm{E}_{\mathrm{c}}\right)$ of the metal. For a large $E_{\mathrm{a}} / E_{\mathrm{c}}$, incoming metal adatoms will have large probability to stick to graphene and the growth will likely be $2 \mathrm{D}$ at low coverage. On the other hand, 3D growth will take place if the ratio $E_{\mathrm{a}} / E_{\mathrm{c}}$ is small since in this case it would be energetically favorable for the metal adatoms to be incorporated into the top of the island instead of sticking to the graphene substrate.

In classical nucleation [100] $n$ determines the diffusion coefficient: low $n$ implies fast diffusion while high $\mathrm{n}$ indicates slow diffusion. Therefore, the island density observed by STM experiment can be correlated to the diffusion barriers of the adatoms on graphene from first-principles calculations.

The $E_{\mathrm{a}} / E_{\mathrm{c}}$ is plotted in Figure 14 as a function of the different adatoms. The diffusion barriers $\Delta E$ have been given in Figure 8b. Liu et al. [71-78] found that almost metals (except alkali metals, Nd and $\mathrm{Sm})$ have an $E_{\mathrm{a}} / E_{\mathrm{c}}$ ratio smaller than 0.5 . In particular, the $E_{\mathrm{a}} / E_{\mathrm{c}}$ ratios for $\mathrm{Cr}, \mathrm{Mn}, \mathrm{Cu}$, and $\mathrm{Au}$ on graphene are extremely small. These results suggest that most of the $3 \mathrm{~d}$ and group 10 transition metals, noble metals, and rare earth metals on graphene will favor a 3D growth mode. In addition, $\mathrm{Cr}, \mathrm{Mn}, \mathrm{Pd}$, $\mathrm{Cu}, \mathrm{Ag}$, and $\mathrm{Au}$ adatoms on graphene exhibit very small diffusion barriers. Therefore, kinetically limited growth would result in relatively large island sizes and small island densities even below room temperature. On the other hand, a high island density and a smaller island size should be expected for other metals on graphene, especially for $\mathrm{Fe}, \mathrm{Co}, \mathrm{Ni}, \mathrm{Pt}$, and $\mathrm{Gd}$. The results of $E_{\mathrm{a}} / E_{\mathrm{c}}$ ratios and diffusion barriers from the calculations can explain some of the features in the growth morphologies observed in the STM experiments as discussed above. The small $E_{\mathrm{a}} / E_{\mathrm{c}}$ ratios for Fe on graphene suggest that these metals will grow in $3 \mathrm{D}$ mode on graphene, consistent with experimental observation (i.e., Figures 5 and 6). The $\mathrm{Pb}$ adatom has extremely low diffusion barrier so that the adatom can move very fast to form the big crystalline islands even at very low temperature (i.e., Figure 7). By contrast, the diffusion barrier of the Fe adatom is much higher so the island density is much higher. By comparing both rare earth metals $\mathrm{Gd}$ and $\mathrm{Eu}$ on graphene, they [72,76] find that Gd exhibits relatively higher diffusion barrier and smaller $E_{\mathrm{a}} / E_{\mathrm{c}}$ ratio (0.36) as compared to those of Eu which has a diffusion barrier of $0.14 \mathrm{eV}$ and a $E_{\mathrm{a}} / E_{\mathrm{c}}$ ratio of 0.50 . The calculation results can also explain why the high density of fractal islands of Gd on graphene. For Eu the diffusion barrier is lower and the $E_{\mathrm{a}} / E_{\mathrm{c}}$ ratio is high which explain why the crystalline islands grow quickly in almost layer-by-layer fashion (i.e., Figure 4). Only at high temperature $(660 \mathrm{~K})$, the growth of Dy can overcome the barrier and grow in bigger crystalline island as shown in Figure 3. However, Gd transforms into hexagonally shaped crystalline islands only when it is heated to a higher temperature $600{ }^{\circ} \mathrm{C}$ (i.e., Figure 2). The growth morphology difference is consistent with the calculated diffusion barriers. 
Figure 14. The ratios of the adsorption energy to the bulk cohesive energy $\left(E_{\mathrm{a}} / E_{\mathrm{c}}\right)$.

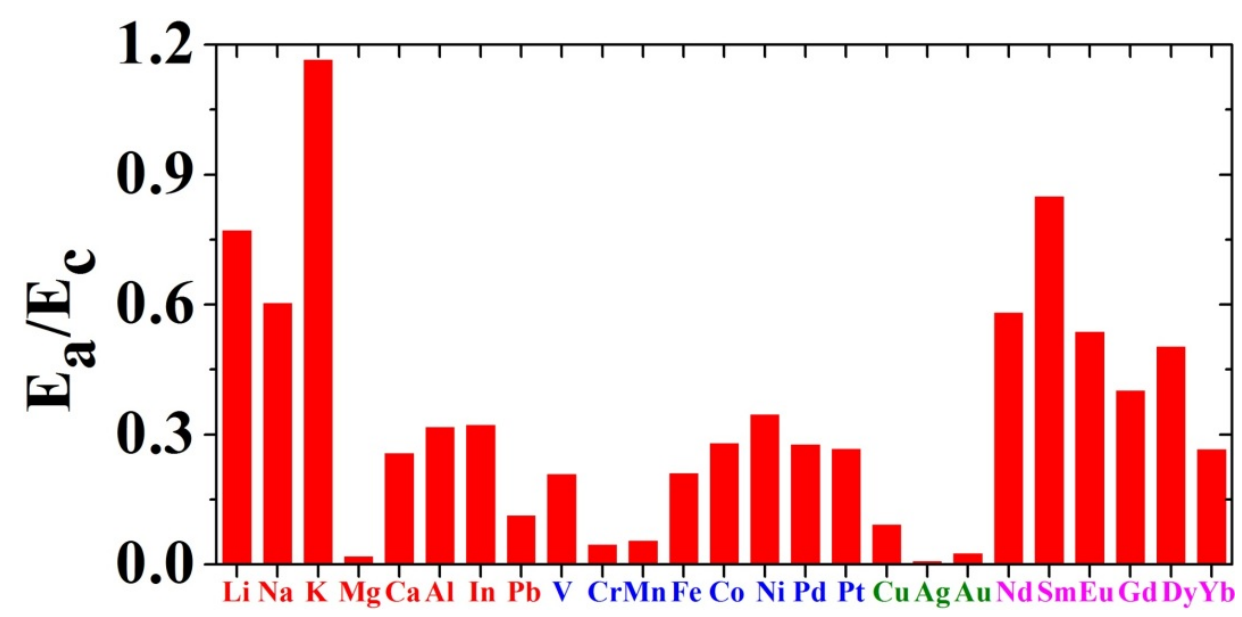

Liu et al. $[72,76]$ note that both Dy and Eu have a very similar $E_{\mathrm{a}} / E_{\mathrm{c}}$ ratio and diffusion barrier $\Delta E$, yet their growth morphologies are quite different. This result suggests that other factors affect the growth morphology in addition to the $E_{\mathrm{a}} / E_{\mathrm{c}}$ ratio and diffusion barrier $\Delta E$. One of the plausible factors affecting the growth morphology besides the $E_{\mathrm{a}} / E_{\mathrm{c}}$ ratio is the interactions between the adatoms as will be discussed in more details in the next section for the case of Fe on graphene. For the case of Dy and $\mathrm{Eu}$, the lattice distortion and electric dipole moment induced by Dy adatom is larger than that induced by Eu adatom. Therefore, stronger repulsive interaction between Dy adatoms it is expected. Attractive interaction between the adatom will promote the big island formation while repulsive interactions will increase the barrier for adatom aggregation and result in smaller islands and higher island density on graphene.

\section{Effects of Adatom Interaction on Growth Morphology}

For practically all metals grown on graphene [71-78] the coverage $\theta$ dependence of $n$ followed classical nucleation attaining steady state at low deposited amounts when the surface area covered is approximately $5 \%$. The constant $n$ at steady state is determined by the condition that the diffusion length becomes equal to the decreasing island separation. However, for Fe growth on graphene an unusual behavior was found by Hupalo et al. [74,77], with $n$ increasing continuously with $\theta$ and surprisingly with very weak $\mathrm{T}$ dependence. This unusual result signifies the presence of long range repulsive interactions between the $\mathrm{Fe}$ adatoms that suppress with increasing $\theta$ the aggregation of the $\mathrm{Fe}$ adatoms to preexisting islands. The magnitude of the repulsive interactions can be extracted from the comparison of the experimental images with kinetic Monte Carlo simulations modeling growth with long range interactions, which were calculated with density functional theory (DFT) [77]. The Fe experiments show how to produce high density of magnetic islands and how this density becomes controllable (by 4 orders of magnitude from $10^{-6}$ to $10^{-2}$ islands $/ \mathrm{nm}^{2}$ ) simply by tuning the deposited Fe amount [74,77].

As discussed in Section 2, the growth morphology for Fe on graphene deviated substantially from the standard expectations. For room temperature deposition, $n$ continues to increase even up to $2.3 \mathrm{ML}$ ( $55 \%$ covered area because the nucleated islands are 3D) and possibly beyond. The island density $n$ is also not sensitive to the deposition temperature. Figure 15 gives more detailed information about island 
heights and sizes $[75,77]$. New islands nucleate, as close as $5 \mathrm{~nm}$ to existing islands. Three different sizes islands, large, medium, and small, are clearly seen from typical line scans, indicating the different times of the ongoing nucleation processes. The large islands do not exceed lateral size of $7 \mathrm{~nm}$. The islands have curved top because of tip convolution. A fraction (40\%) of newly arriving $\mathrm{Fe}$ atoms diffuses to the large islands and moves to higher layers, while a larger fraction $(60 \%)$ nucleates new islands. The diffusion length measured at the lower $\theta=0.003 \mathrm{ML}$ is at least 10 times larger than $5 \mathrm{~nm}$, the average distance from preexisting islands at which new islands nucleate. So apparently there is a barrier that prohibits the adatoms to attach to the islands already present. Encountering another diffusion adatom, that was also repelled, results in the nucleation of a new island, so the island density continues to grow with $\theta$.

Figure 15. Detailed image of the structure and distribution of Fe islands. (a) The area is $100 \times 100 \mathrm{~nm}^{2}, \mathrm{n}=7 \times 10^{-3}$ islands $/ \mathrm{nm}^{2}, \theta=0.6 \mathrm{ML}, \mathrm{T}=450 \mathrm{~K}$, and $\mathrm{F}=5.33 \times 10^{-2} \mathrm{ML} / \mathrm{min}$. New islands nucleate at a distance as close as $5 \mathrm{~nm}$ to preexisting islands; (b) Three different size islands are seen from the image and the line scan.
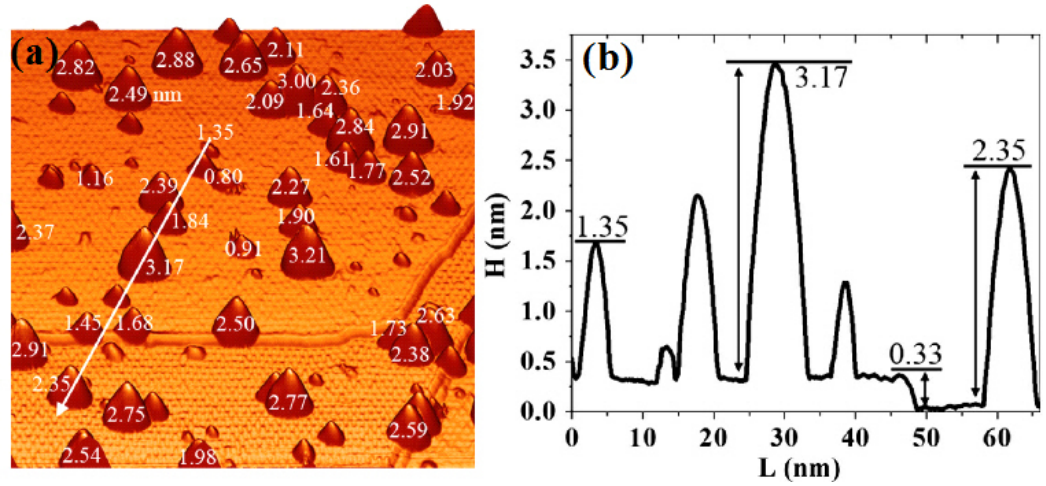

There are three possible mechanisms through which adatoms on graphene interact as Liu et al. suggested [75,77]. One is the long-range interaction originating from the indirect (or RKKY) interaction mediated through the delocalized electrons on graphene due to Friedel oscillation [108-113]. The strength of such an interaction will be dependent on the density of states of the free electrons on the graphene. According to the bonding electron distribution as shown in Figures 9 and 10, the indirect interactions would be stronger between alkali and simple metal adatoms but smaller for 3d-transiton metal adatoms because the electrons in the 3d-transition metal/graphene systems are much more localized. The indirect interaction between the rare earth metal adatoms would be intermediate between the above two cases.

Another possible interaction as suggested by Liu et al. [75] is the electric dipole-dipole interaction between the adatoms since most of the metal adatoms on graphene induce electric dipole moment. Such interaction is repulsive and proportional to the magnitude of the dipole moment and inverse proportional to the cubic power of the separation distance between the adatoms. Alkali adatoms ( $\mathrm{Li}$, $\mathrm{Na}$, and $\mathrm{K}$ ) as well as In and $\mathrm{Nd}$ have large electric dipole moment and should feel stronger repulsion due to the dipole-dipole interaction. On the other hand, dipole-dipole interaction between $\mathrm{Mg}, \mathrm{Cu}, \mathrm{Pb}$, $\mathrm{Mn}, \mathrm{Cr}, \mathrm{Ag}$, and Pt should be small. 
Finally, there also ought to be elastic interaction between the adatoms mediated through graphene because of the adsorption induced graphene lattice distortion [75]. The repulsion strength of such interaction should be proportional to the distortion amplitude caused by the adatoms. In this regard, most of the 3d-transition metal adatoms (especially $\mathrm{Fe}, \mathrm{Co}, \mathrm{Pd}$, and $\mathrm{Pt}$ ) should feel stronger elastic repulsion than the others.

To gain insight into the unusual Fe growth morphology observed in the STM experiments, first principles calculations to study the interaction between Fe adatoms on graphene have been carried out $[75,77]$. The interaction energy between the two adatoms on graphene defined as $E_{\text {inter }}(\mathrm{r})=E_{\mathrm{a} 2}(\mathrm{r})-2 E_{\mathrm{a} 1}$, where $E_{\mathrm{a} 2}(\mathrm{r})$ is the adsorption energy of two $\mathrm{Fe}$ adatoms on graphene at separation $r$, and $E_{\mathrm{a} 1}$ is the adsorption energy of a single Fe adatom, has been calculated. Figure 16 shows $E_{\text {inter }}(r)$ as a function of $r$ between the two adatoms. It is interesting to note that the interaction between $\mathrm{Fe}-\mathrm{Fe}$ adatoms is attractive at small separations (less than about $4.0 \AA$ ) but becomes repulsive at larger distances greater than $5.0 \AA$ from the studies of Liu et al. [75,77]. The repulsive interactions for Fe adatoms at larger distances can account for the unusual nucleation of Fe islands on graphene. The diffusion length of $\mathrm{Fe}$ adatoms at lower coverage $(\sim 0.003 \mathrm{ML})$ and at a temperature of $450 \mathrm{~K}$ estimated from the experimentally observed island density based on the scaling theory of nucleation [96] would be at least 50 times larger than $5 \mathrm{~nm}$, where newly formed islands are seen to nucleate (see Figure 15a). If the only controlling barriers are terrace diffusion and aggregation to the islands, there is no reason for the diffusion adatom not to join the island. Therefore, apparently there is a barrier that prohibits adatoms from approaching the islands already present, and the adatoms continue to diffuse on the vacant part of the surface, even very close to the preexisting islands. Encountering another adatom that was also repelled from preexisting islands results in ongoing nucleation of new islands. The origin of this repulsive barrier is related to the Fe-Fe repulsive interaction calculated above.

Figure 16. Interaction energy $E$ (red large open circle symbols) and $V(r)$ (blue solid line) of $\mathrm{Fe}-\mathrm{Fe}$ adatoms on graphene as a function of $\mathrm{Fe}-\mathrm{Fe}$ separation. $E_{\mathrm{e}}$ (small open symbols) and $V_{\mathrm{d}}(\mathrm{r})$ (dash line) are elastic interaction and dipole-dipole interaction, respectively.

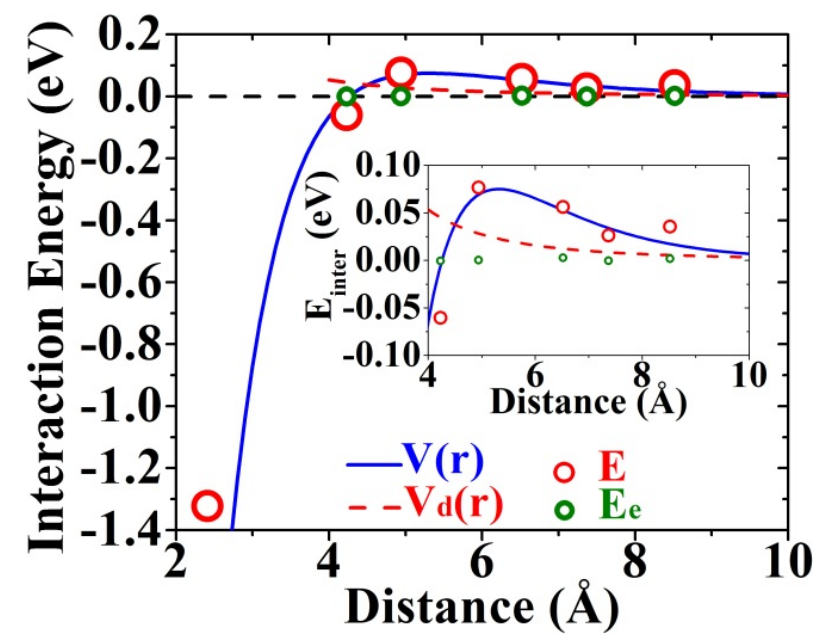

Whereas the strong attractive interaction between the Fe adatoms at small separation is due to metallization effects, the origin of the repulsive interaction at larger distances is more complex. The 
first-principles calculation results by Liu et al. [75] shows that electric dipole-dipole interaction can contribute only part of the repulsive force $(\sim 30 \%)$ between Fe adatoms, and the repulsion due to the elastic interaction is small. It is suggested that the dominant contribution to the repulsive energy would originate from the indirect (or RKKY) interactions mediated through the delocalized electrons on graphene.

\section{Thermal Stability of Metal Nanostructures on Graphene}

Graphene has been used as substrate for surface-supported catalysis [65-70]. Enhanced electrocatalytic activity of $\mathrm{Pt}, \mathrm{Pd}$, and $\mathrm{PdRu}$ nanoparticles on graphene has been reported by several experimental groups [65-70]. It has also been shown that Pt nanoparticles on graphene are very stable against aggregation among them or desorption from the graphene substrate. Nevertheless, the mechanism underlying the enhanced catalytic activity and durability of the metal nanostructures on graphene is still not well understood. In general three-dimensional (3D) metal nanostructures with high density and high thermal stability against coarsening and desorption are desirable for surface-supported catalysis applications. In Sections 2 and 5, we have shown that most of metals on graphene grown on Si terminated $\mathrm{SiC}$ substrate will favor 3D island growth morphology. Knowledge of the thermal stability for metal nanostructures on graphene would also be an essential step towards a fundamental understanding of their catalytic performance.

In order to provide some insights into the thermal stability of the metal nanoislands on graphene, Liu et al. [76,78] have calculated the difference between the bulk cohesive energy and adsorption energy of the metals on graphene, i.e., $E_{\mathrm{c}}-E_{\mathrm{a}}$ for various metals adatoms on graphene. The results are plotted in Figure 17. They proposed that such an energy difference is the dominant factor that sets the temperature for the metal atoms to detach from the islands to the graphene layer in thermodynamics limit. For a metal with small $E_{\mathrm{c}}-E_{\mathrm{a}}$, coarsening of the metal islands on graphene will easily take place because atoms can be easily detached from the small islands and diffuse on the top of graphene to join other bigger islands. On the other hand, larger $E_{\mathrm{c}}-E_{\mathrm{a}}$ will require higher temperature for island coarsening and the metal islands will be much more stable.

Figure 17. Energy difference $\left(E_{\mathrm{c}}-E_{\mathrm{a}}\right)$ between the bulk cohesive energy $\left(E_{\mathrm{c}}\right)$ and the adsorption energy $\left(E_{\mathrm{a}}\right)$.

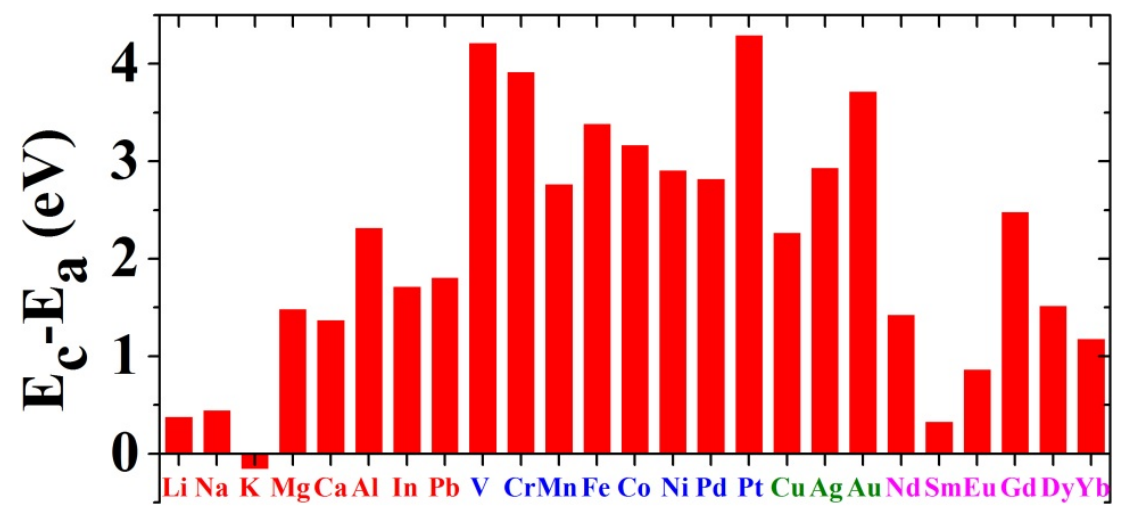

As shown in Figure 17, coarsening of alkali metal and some rare earth metal ( $\mathrm{Sm}$ and $\mathrm{Eu}$ ) islands will take place at much lower temperatures. On the other hand, the 3d-transiton metal, group 
10 transition metals and noble metals exhibit much higher thermal stability against coarsening. The thermal stabilities of the group III and IV metal islands and other rare earth metal islands on graphene are lower than that of the 3d-transition metals, group 10 transition metals, and noble metals but should be much higher than the alkali metals. The thermal stability of the metal nanostructures is desirable for surface-supported catalysis applications. Their study suggests that graphene supported 3d-transition metals, group 10 transition metals and noble metal nanostructures should be a good system for supported catalysis.

The predictions from the first-principles calculations are confirmed by experiments [76,78]. Using STM, thermal stability of several metals ( $\mathrm{Fe}, \mathrm{Gd}, \mathrm{Dy}$, and $\mathrm{Eu}$ ) on graphene have been investigated. The metals were initially deposited at room temperature (RT) on graphene prepared by thermal annealing of $\mathrm{SiC}$ [91]. After the characterization of the growth the metal islands were annealed to a higher temperature. The island morphologies before and after annealing measured by STM are compared [78].

Figure $18 \mathrm{a}$ shows the Fe island morphology at a coverage of $\theta=3.0 \mathrm{ML}$, RT deposition with a flux of $\mathrm{F}=0.25 \mathrm{M} / \mathrm{min}$. The island density is $\mathrm{N}=5.1 \times 10^{-3}$ islands $/ \mathrm{nm}^{2}$ after RT deposition. Annealing at $660 \mathrm{~K}$ for $20 \mathrm{~min}$ from Figure 18a changes the islands density only slightly, the shapes of individual islands change due to equilibration, as shown in Figure 18b. In particular, the islands grow in height as seen from the 1D line scan of the islands before and after annealing shown in Figure 19a. This is a unique feature of metal growth on graphene and very useful result for potential catalytic applications [74,78].

For Gd growth on graphene, fractal-like morphology [72,74,78] is observed with RT deposition even at a high coverage of 4.0 ML, as shown in Figure 18c. The fractal-like morphology is stable up to $800 \mathrm{~K}$ but a transition to crystalline shapes occurs at higher annealing temperature. This is shown in Figure $18 \mathrm{~d}$ where the film at Figure $18 \mathrm{c}$ was annealed at $1050 \mathrm{~K}$ for a short time $(5 \mathrm{~min})$. The island height histograms indicate that the islands also grow in height after annealing but the island density is similar (within 10\%) before and after annealing. The larger relative increase in height vs. size upon annealing can be seen from the 1D line scan as shown in Figure 19b.

As reported by Liu et al. and Hupalo et al. [72,74], the growth of Dy on graphene is somewhat different from $\mathrm{Fe}$ and $\mathrm{Gd}$ on graphene. 2.0 $\mathrm{ML}$ of Dy deposited on graphene at $\mathrm{RT}$ with $\mathrm{F}=0.22 \mathrm{ML} / \mathrm{min}$ results in island density $\mathrm{N}=6 \times 10^{-3}$ islands $/ \mathrm{nm}^{2}$ as shown in Figure 8e. Annealing to $580 \mathrm{~K}$ transforms the multiple level islands (4-7 Dy layers) into more uniform height (6 and 7 layers) islands and some of the islands are found to coalesce (Figures $18 \mathrm{f}$ and 19c). These results indicate lower thermal stability of Dy nanoparticles on graphene, as compared with Fe and Gd. The 1D line scan in Figure 19 not only show the larger relative increase in height vs. size (higher aspect ratios) upon annealing, but also show that the islands form more uniform height islands after annealing. Dy is the metal with the least amount of changes as seen in Figure 19c and the islands always have nonuniform tops before and after annealing, indicating unusual nucleation sites when the Dy islands nucleate initially.

By contrast, experiment showed the growth morphology of Eu on graphene is very different from that of $\mathrm{Fe}, \mathrm{Gd}$, and Dy [72,78]. 2.6 ML of Eu deposited on graphene at RT with $\mathrm{F}=0.16 \mathrm{ML} / \mathrm{min}$ exhibits connected and large islands as shown in Figure 18g. Annealing the Eu film seen in Figure $18 \mathrm{~g}$ at $365 \mathrm{~K}$ for $15 \mathrm{~min}$ transforms the large islands into almost continuous films with thickness of $3 \mathrm{ML}$ as one can see from Figure $18 \mathrm{~h}$. 
Figure 18. The morphologies and thermal stability of high coverage $\mathrm{Fe}, \mathrm{Gd}$, and $\mathrm{Eu}$ on graphene. (a) $250 \times 250 \mathrm{~nm}^{2}$ area after Fe deposition on graphene at RT with $\theta=3 \mathrm{ML}$, $\mathrm{F}=0.25 \mathrm{ML} / \mathrm{min}$. The island density is $\mathrm{N}=5.1 \times 10^{-3}$ islands $/ \mathrm{nm}^{2}$; (b) Annealing at $600 \mathrm{~K}$ for $20 \mathrm{~min}$ from (a) does not change island density significantly, only the shape of individual islands equilibrates and their average height increases; (c) Fractal-like morphology of $4 \mathrm{ML} \mathrm{Gd}$ on graphene grown at RT in $250 \times 215 \mathrm{~nm}^{2}$ area, $\mathrm{F}=0.1 \mathrm{ML} / \mathrm{min}$. Fractal morphology is stable up to $800 \mathrm{~K}$, but transition to crystalline shapes occurs at higher temperature, as shown in (d) where previous film was annealed at $1050 \mathrm{~K}$ for $5 \mathrm{~min}$; (e) $2.0 \mathrm{ML}$ of Dy deposited on graphene at RT, image area $100 \times 100 \mathrm{~nm}^{2}, \mathrm{~F}=0.22 \mathrm{ML} / \mathrm{min}$, island density $\mathrm{N}=6 \times 10^{-3}$ islands $/ \mathrm{nm}^{2}$; (f) annealing of (e) to $580 \mathrm{~K}$ transforms multiple level islands with 4-7 Dy layers exposed into more uniform height shaped islands and some of them coalesce, indicating lower thermal stability of Dy nanoparticles on graphene, as compared with Fe and Gd; (g) 2.6 ML of Eu deposited on graphene at RT, image area $250 \times 200 \mathrm{~nm}^{2}, \mathrm{~F}=0.16 \mathrm{ML} / \mathrm{min}$; (h) Annealing of the $\mathrm{Eu}$ film from $(\mathbf{g})$ at $365 \mathrm{~K}$ for $15 \mathrm{~min}$ transforms the lager islands into almost continuous film with thickness of $3 \mathrm{ML}$.
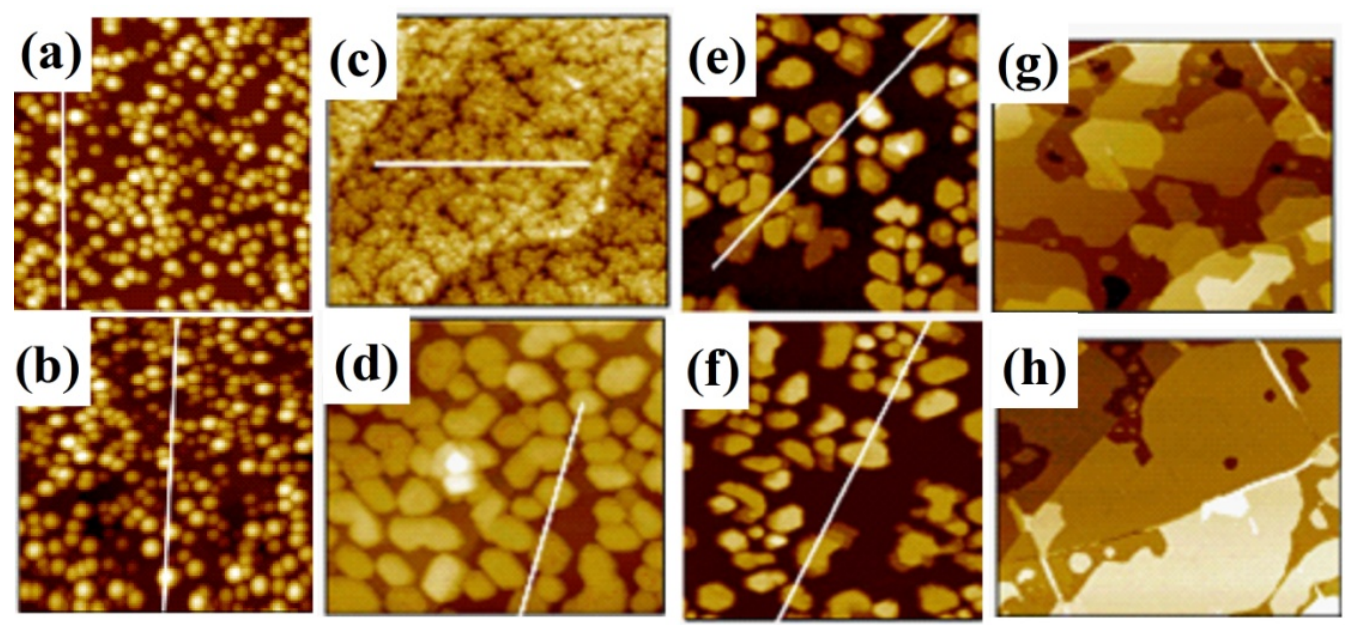

Figure 19. 1D scans along the lines shown in Figure 18 for three of the metals (a) Fe; (b) Gd; and (c) Dy. One line (dash black) is for RT the other (solid red) is after annealing to the higher temperature. The aspect ratio (height/width) increases with annealing which will enhance the fraction of low coordination sites and the catalytic activity with increasing temperature. Both height and width grow, but the relative height increase is larger than the relative width increase.
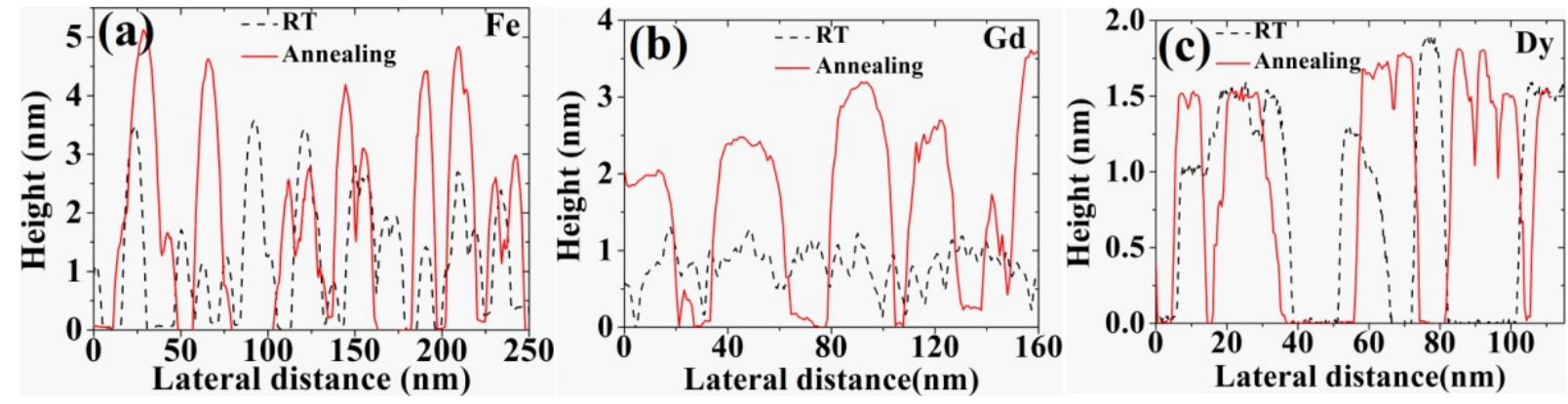


\section{Summary}

In this article, we summarized experimental and first-principles calculations studies of adsorption, interaction, growth morphology, and thermal stability for metal on graphene on $6 \mathrm{H}-\mathrm{SiC}(0001)$ substrate. We showed that different metals on graphene exhibit very different growth morphologies and thermal stability depending on their interaction with graphene and interaction among the metal adatoms. We also showed that there is a good correlation between the adsorption energy, diffusion barrier and bond order for 3d-transion metals, group 10 transition metals, noble metals, and rare earth metals on graphene. For metal adatoms that have larger bond order values on graphene, their adsorption energies and diffusion barriers are also larger (except Pd). This trend can be attributed to various degrees of covalent bonding mainly from the d-electrons of the metal adatoms. However, the correlation between the adsorption strength and the value of the bond order is not obvious for group I-IV metals on graphene. The group I alkali metal adatoms on graphene have essentially a zero bond order value. This is because the s electron of the alkali metals can be easily transferred to graphene and the interaction between the alkali adatom and graphene is mainly ionic. For group II metal on graphene like $\mathrm{Mg}$, the $3 \mathrm{~s}^{2}$ electrons bond tightly to its core, resulting in physisorption of $\mathrm{Mg}$ on graphene. In contrast, the $4 \mathrm{~s}^{2}$ electrons of the $\mathrm{Ca}$ adatom are found to be transferred substantially to graphene due to their larger separation from the Ca nucleus and lead to stronger ionic bonding between the $\mathrm{Ca}$ adatom and graphene thus to larger adsorption energy and diffusion barrier. The group III and IV metals on graphene exhibit a mixture of ionic and covalent bonds, from $\mathrm{Al}$, In to $\mathrm{Pb}$, suggesting that the ionic bonding is still a dominating factor in these systems.

We also showed that the growth morphology of metals on graphene can be affected by several parameters. In general, the ratios of the adsorption energy to the bulk cohesive energy $\left(E_{\mathrm{a}} / E_{\mathrm{c}}\right)$ and the diffusion barrier $(\Delta E)$ can account for the growth morphology of metals on graphene. The $E_{\mathrm{a}} / E_{\mathrm{c}}$ ratio determines whether the islands will grow in $2 \mathrm{D}$ or $3 \mathrm{D}$ mode. Adatoms which have a small $E_{\mathrm{a}} / E_{\mathrm{c}}$ ratio will prefer a $3 \mathrm{D}$ growth mode and for lower $\Delta E$ the $3 \mathrm{D}$ will be more pronounced. The diffusion barrier controls the density of the islands, the higher the barrier, the higher the island density. Long range interaction such as indirect electron-electron interaction mediated through the free electrons on graphene, dipole-dipole interaction, and elastic interaction can affect the energy landscape and diffusion barrier for the growth and can also play an important role in the observed growth morphology. Our calculation results also indicate that most of the 3d-transition metals, group 10 transition metals, noble metals, and some rare earth metal nanostructures formed on graphene exhibit high stability against coarsening. This remarkable property makes the graphene-supported metal nanostructures a desirable system for catalysis applications.

For practically all metals the grown islands are $3 \mathrm{D}$ as a result of the low ratio of the metal adsorption energy on graphene to the metal cohesive energy. Only Eu grows in large interconnected layers covering graphene. The growth of Fe on graphene is also unusual because it does not follow classical nucleation. The nucleated island density is unexpectedly high, it increases continuously with deposited amount $\theta$ and shows no temperature dependence [74,77,78]. These unexpected results indicate the presence of long range repulsive interactions which are confirmed with kinetic Monte Carlo simulations and DFT calculations [77]. The island density becomes tunable with $\theta$ which can be useful in magnetic storage applications. The growth of Dy islands up to $700 \mathrm{~K}$ shows ideal triangular 
instead of hexagonal shapes expected for hcp(0001) crystals which is currently under investigation most likely suggesting that the Dy islands have fcc(111) and not $h c p(0001)$ structure. This can have importance implications about the electronic and magnetic properties of the islands.

Thermal annealing of the metal islands shows limited coarsening in almost all cases with the island density almost unchanged but the aspect ratio (height/ lateral size) increasing which can be relevant for catalysis [78].

Finally, we would like to point out that our calculations are performed on a free standing graphene sheet. Effects of the substrate should be considered when the calculation results are used to interpret experimental data. For graphene on metal surfaces, it has been shown that the substrate effects can be significant. However, for graphene on the Si-terminated SiC surface as in the case of our present experiments, the interaction between the graphene and the substrate is weak and can be neglected to a good approximation.

\section{Acknowledgments}

Work at Ames Laboratory was supported by the US Department of Energy, Basic Energy Sciences, Division of Materials Science and Engineering, including a grant of computer time at the National Energy Research Supercomputing Center (NERSC) in Berkeley, CA under Contract No. DE-AC02-07CH11358. Xiaojie Liu also acknowledges the support by the National Natural Science Foundation of China under Grant No. 11204013.

\section{References}

1. Novoselov, K.S.; Geim, A.K.; Morozov, S.V.; Jiang, D.; Zhang, Y.; Dubonoset, S.V.; Grigorieva, I.V.; Firsov, A.A. Electric field effect in atomically thin carbon films. Science 2004, 306, 666-669.

2. Novoselov, K.S.; Geim, A.K.; Morozov, S.V.; Jiang, D.; Katsnelson, M.I.; Grigorieva, I.V.; Dubonos, S.V.; Firsov, A.A. Two-dimensional gas of massless Dirac fermions in graphene. Nature 2005, 438, 197-200.

3. Zhang, Y.; Tan, Y.W.; Stormer, H.L.; Kim, P. Experimental observation of the quantum Hall effect and Berry's phase in graphene. Nature 2005, 438, 201-204.

4. Balandin, A.A.; Ghosh, S.; Bao, W.; Calizo, I.; Teweldebrhan, D.; Miao, F.; Lau, C.N. Superior thermal conductivity of single-layer graphene. Nano Lett. 2008, 8, 902-907.

5. Lee, C.; Wei, X.; Kysar, J.W.; Hone, J. Measurement of the elastic properties and intrinsic strength of monolayer graphene. Science 2008, 321, 385-388.

6. Castro Neto, A.H.; Guinea, F.; Peres, N.M.R.; Novoselov, K.S.; Geim, A.K. The electronic properties of graphene. Rev. Mod. Phys. 2009, 81, 109-162.

7. Geim, A.K.; Novoselov, K.S. The rise of graphene. Nat. Mater. 2007, 6, 183-191.

8. Das Sarma, S.; Adam, S.; Hwang, E.H.; Rossi, E. Electronic transport in two-dimensional graphene. Rev. Mod. Phys. 2011, 83, 407-470. 
9. Seneor, P.; Dlubak, B.; Martin, M.B.; Anane, A.; Jaffres, H.; Fert, A. Spintronics with graphene. MRS Bull. 2012, 37, 1245-1254.

10. Song, C.L.; Sun, B.; Wang, Y.L.; Jiang Y.P.; Wang, L.; He, K.; Chen, X.; Zhang P.; Ma, X.C.; Xue, Q.K. Charge-transfer-induced cesium superlattices on graphene. Phys. Rev. Lett. 2012, 108, 156803:1-156803:5.

11. Virojanadara, C.; Watcharinyanon, S.; Zakharov, A.A.; Johansson, L.I. Epitaxial graphene on 6H-SiC and Li intercalation. Phys. Rev. B 2010, 82, 205402:1-205402:6.

12. Caragiu, M.; Finberg, S. Alkali metal adsorption on graphite: A review. J. Phys. Condens. Matter 2005, 17, R995-R1024.

13. Ferralis, N.; Pussi, K.; Finberg, S.E.; Smerdon, J.; Lindroos, M.; McGrath, R.; Diehl, R.D. Low-energy electron diffraction study of potassium adsorbed on single-crystal graphite and high oriented pyrolytic graphite. Phys. Rev. B 2004, 70, 245407:1-245407:6.

14. Renard, J.; Lundeberg, M.B.; Folk, J.A.; Pennec, Y. Real-time imaging of K atoms on graphite: Interactions and diffusion. Phys. Rev. Lett. 2011, 106, 156101:1-156101:4.

15. Knudsen, J.; Feibelman, P.J.; Gerber, T.; Grånäs, E.; Schulte, K.; Stratmann, P.; Andersn, J.N.; Michely, T. Clusters binding to the graphene moiré on $\operatorname{Ir}(111)$ : X-ray photoemission compared to density functional calculations. Phys. Rev. B 2012, 85, 035407:1-035407:6.

16. N'Diaye, A.T.; Gerber, T.; Busse, C.; Mysliveček, J.; Coraux, J.; Michely, T. A versatile fabrication method for cluster superlattices. New J. Phys. 2009, 11, 103045:1-103045:19.

17. Zhou, Z.; Gao, F.; Goodman, D.W. Deposition of metal clusters on single-layer graphene/Ru(0001): Factors that govern cluster growth. Surf. Sci. 2010, 604, L31-L38.

18. Park, S.; Shao, Y.; Wan, H.; Rieke, P.C.; Viswanathan, V.V.; Towne, S.A.; Saraf, L.V.; Liu, J.; Lin, Y.; Wang, Y. Design of graphene sheets-supported Pt catalyst layer in PEM fuel cells. Electrochem. Commun. 2011, 13, 258-261.

19. Cretu, O.; Rodríguez-Manzo, J.A.; Demortière, A.; Banhart, F. Electron beam-induced formation and displacement of metal clusters on graphene, carbon nanotubes and amorphous carbon. Carbon 2012, 50, 259-264.

20. Pi, K.; McCreary, K.M.; Bao, W.; Han, W.; Chiang, Y.F.; Li, Y.; Tsai, S.W.; Lau, C.N.; Kawakami, R.K. Electronic doping scattering by transition metals on graphene. Phys. Rev. B 2009, 80, 075406:1-075406:5.

21. Zan, R.; Bangert, U.; Ramasse, Q.; Novoselov, K.S. Evolution of gold nanostructures on graphene. Small 2011, 20, 2868-2872.

22. Xu, Y.; Semidey-Flecha, L.; Liu, L.; Zhou, Z.; Goodman, D.W. Exploring the structure and chemical activity of 2-D gold islands on graphene moiré/Ru(0001). Faraday Discuss. 2011, 152, 267-276.

23. Aton, R.; Kreutzer, P. In situ TEM evaluation of the growth kinetics of Au pariticles on highly oriented pyrolithic graphite at elevated temperatures. Phys. Rev. B 2000, 61, 16077-16083.

24. Zan, R.; Bangert, U.; Ramasse, Q.; Novoselov, K.S. Metal-graphene interaction studied via atomic resolution scanning transmission electron microscopy. Nano Lett. 2011, 11, 1087-1092.

25. Liu, L.; Zhou, Z.; Guo, Q.; Yan, Z.; Yao, Y.; Goodman, D.W. The 2-D growth of gold on single-layer graphene/Ru(0001): Enhancement of CO adsorption. Surf. Sci. 2011, 605, L47-L50. 
26. Wong, P.K.J.; de Jong, M.P.; Leonardus, L.; Siekman, M.H.; van der Wiel, W.G. Growth mechanism and interface magnetic properties of Co nanostructures on graphite. Phys. Rev. B 2011, 84, 054420:1-054420:5.

27. Vo-van, C.; Schumacher, S.; Coraux, J.; Sessi, V.; Fruchart, O.; Brookes, N.B.; Ohresser, P.; Michely, T. Magnetism of cobalt nanoclusters on graphene on iridium. Appl. Phys. Lett. 2011, 99, 142504:1-142504:3.

28. Vo-van, C.; Kassir-Bodon, Z.; Yang, H.; Coraux, J.; Vogel, J.; Pizzini, S.; Bayle-Guillemaud, P.; Chshiev, M.; Ranno, Laurent, Guisset, V.; David, P.; Salvador, V.; Fruchart, O. Ultrathin epitaxial cobalt films on graphene for spintronic investigations and applications. New J. Phys. 2010, 12, 103040:1-103040:15.

29. Förster, D.F.; Wehling, T.O.; Schumacher, S.; Rosch, A.; Michely, T. Phase coexistence of clusters and islands: Europium on graphene. New J. Phys. 2012, 14, 023022:1-023022:26.

30. Sutter, E.; Albrecht, P.; Wang, B.; Bocquet, M.-L.; Wu, L.; Zhu, Y.; Sutter, P. Arrays of Ru nanoclsuters with narrow size distribution template by monolayer graphene on Ru. Surf. Sci. 2011, 605, 1676-1684.

31. Rytkönen, K.; Akola, J.; Manninen, M. Density functional study of alkali-metal atoms and monolayers on graphite (0001). Phys. Rev. B 2007, 75, 075401:1-075401:9.

32. Uthaisar, C.; Barone, V.; Peralta, J.E. Lithium adsorption on zigzag graphene nanoribbons. $J$. Appl. Phys. 2009, 106, 113715:1-113715:6.

33. Jin, K.H.; Choi, S.M.; Jhi, S.H. Crossover in the adsorption properties of alkali metals on graphene. Phys. Rev. B 2010, 82, 033414:1-033414:4.

34. Lugo-Solis, A.; Vasiliev, I. Ab initio study of $\mathrm{K}$ adsorption on graphene and carbon nanotubes: Role of long-range ionic force. Phys. Rev. B 2007, 76, 235431:1-235431:8.

35. Martínez, J.I.; Cabria, I.; López, M.J.; Alonso, J.A. Adsorption of lithium on finite graphitic clusters. J. Phys. Chem. C 2009, 113, 939-941.

36. Davydov, S.Y. Adsorption of Sodium and cesium on graphite: Work function calculations. Tech. Phys. Lett. 2009, 35, 998-1000.

37. Valencia, F.; Romero, A.H.; Ancilotto, F.; Silvestrelli, P.L. Lithium adsorption on graphite from density functional theory calculations. J. Phys. Chem. B 2006, 110, 14832-14841.

38. Chan, K.T.; Neaton, J.B.; Cohen, M.L. First-principles study of metal adatom adsorption on graphene. Phys. Rev. B 2008, 77, 235430:1-235430:12.

39. Sanchez-Paisal, Y.; Sanchez-Portal, D.; Ayuela, A. Ab initio calculations of zirconium adsorption and diffusion on graphene. Phys. Rev. B 2009, 80, 045428:1-045428:10.

40. Mao, Y.; Yuan, J.; Zhong, J. Density functional calculation of transition metal adatom adsorption on graphene. J. Phys. Condens. Matter 2008, 20, doi:10.1088/0953-83-8984/20/11/115209.

41. Sevinçli, H.; Topsakal, M.; Durgun, E.; Ciraci, S. Electronic and magnetic properties of 3d transition metal atom adsorbed graphene and graphene nanoribbons. Phys. Rev. B 2008, 77, 195434:1-195434:7.

42. Zanella, I.; Fagan, S.B.; Mota, R.; Fazzio, A. Electric and magnetic properties of Ti and Fe on graphene. J. Phys. Chem. C 2008, 112, 9163-9168. 
43. Zólyomi, V.; Rusznyák, Á.; Kürti, J.; Lambert, C.J. First principles study of the binding of 4d and 5d transition metals to graphene. J. Phys. Chem. C 2010, 114, 18548-18552.

44. Wehling, T.O.; Balatsky, A.V.; Katsnelson, M.I.; Lichtenstein, A.I.; Rosch, A. Orbitally controlled Kondo effect of Co adatoms on grapheme. Phys. Rev. B 2010, 81, 115427:1-115427:6.

45. Porter, C.D.; Stroud, D. Clustering and magnetic anisotropy of Fe adatoms on graphene. Phys. Rev. B 2012, 85, 235452:1-235452:10.

46. Nemec, N.; Tománek, D.; Cuniberti, G. Contact dependence of carrier injection in carbon nanotubes: An ab initio study. Phys. Rev. Lett. 2006, 96, 076802:1-076802:4.

47. Krasheninnikov, A.V.; Lehtinen, P.O.; Foster, A.S.; Pyykkö, P.; Nieminen, R.M. Embedding transition-metal atoms in graphene: Structure, bonding, and magnetism. Phys. Rev. Lett. 2009, 102, 126807:1-126807:4.

48. Cao, C.; Wu, M.; Jiang, J.; Cheng, H.P. Transition metal adatom and dimer adsorbed on graphene: Induced magnetization and electronic structures. Phys. Rev. B 2010, 81, 205424:1-205424:9.

49. Ishii, A.; Yamamoto, M.; Asano, H.; Fujiwara, K. DFT calculation for adatom adsorption on graphene sheet as a prototype of carbon nano tube functionalization. J. Phys. Conf. Ser. 2008, 100, 052087:1-052087:4.

50. Longo, R.C.; Carrete, J.; Ferrer, J.; Gallego, L.J. Structural, magnetic, and electronic properties of $\mathrm{Ni}_{n}$ and $\mathrm{Fe}_{n}$ nanostructures $(\mathrm{n}=1-4)$ adsorbed on zigzag nanoribbons. Phys. Rev. B 2010, 81, 115418:1-115418:10.

51. Johll, H.; Kang, H.C. Density functional theory of Fe, Co, and Ni adatoms and dimmers adsorbed on graphene. Phys. Rev. B 2009, 79, 245416:1-245416:18.

52. Duffy, D.M.; Blackman, J.A. Magnetism of $3 \mathrm{~d}$ transition-metal adatoms and dimmers on graphite. Phys. Rev. B 1998, 58, 7443-7449.

53. Wu, M.; Liu, E.Z.; Ge, M.Y.; Jiang, J.Z. Stability, electronic, and magnetic behaviors of $\mathrm{Cu}$ adsorbed graphene: A first-principles study. Appl. Phys. Lett. 2009, 94, 102505:1-102505:3.

54. Aktürk, O.Ü.; Tomak, M. $\mathrm{Au}_{n} \mathrm{Pt}_{n}$ clusters adsorbed on graphene studied by first-principles calculations. Phys. Rev. B 2009, 80, 085417:1-085417:6.

55. Amft, M.; Sanyal, B.; Eriksson, O.; Skorodumova, N.V. Small gold clusters on graphene, their mobility and clustering: A DFT study. J. Phys. Condens. Matter 2011, 23, doi:10.1088/0953-8984/23/20/205301.

56. Varns, R.; Strange, P. Stability of gold atoms and dimers adsorbed on graphene. J. Phys. Condens. Matter 2008, 20, 225005:1-225005:8.

57. Okazaki-Maeda, K.; Morikawa, Y.; Tanaka, S.; Kohyama, M. Structures of Pt clusters on graphene by first-principles calculations. Surf. Sci. 2010, 604, 144-154.

58. Zhou, Y.G.; Zu, X.T.; Gao, F.; Lv, H.F.; Xiao, H.Y. Adsorption-induced magnetic properties and metallic behavior of graphene. Appl. Phys. Lett. 2009, 95, 123119:1-123119:3.

59. Balog, R.; Jørgensen, B.; Wells, J.; Lægsgaard, E.; Hofmann, P.; Besenhacher, F.; Hornekær, L. Atomic hydrogen adsorbate structures on graphene. J. Am. Chem. Soc. 2009, 131, 8744-8745.

60. Li, W.; Zhao, M.; Xia, Y.; Zhang, R.; Mu, Y. Covalent-adsorption induced magnetism in graphene. J. Mater. Chem. 2009, 19, 9274-9282. 
61. Ivanovskaya, V.V.; Zobelli, A.; Teillet-Billy, D.; Rougeau, N.; Sidis, V.; Briddon, P.R. Hydrogen adsorption on graphene: A first principles study. Eur. Phys. J. B 2010, 76, 481-486.

62. Wehling, T.O.; Katsnelson, M.I.; Lichtenstein, A.I. Impurities on graphene: Midgap states and migration barriers. Phys. Rev. B 2009, 80, 085428:1-085428:7.

63. Wang, W.X.; liang, S.H.; Yu, T.; Li, D.H.; Li, Y.B.; Han, X.F. The study of interaction between graphene and metals by Raman spectroscopy. J. Appl. Phys. 2011, 109, 07C501:1-07C501:3.

64. Khomyakov, P.A.; Giovannetti, G.; Rusu, P.C.; Brocks, G.; van den Brink, J.; Kelly, P.J. First-principles study of the interaction and charge transfer between graphene and metals. Phys. Rev. B 2009, 79, 195425:1-195425:12.

65. Yoo, E.J.; Okata, T.; Akita, T.; Kohyama, M.; Nakamura, J.; Honma, I. Enhanced electrocatalytic activity of Pt subnanoclusters on graphene nanosheet surface. Nano Lett. 2009, 9, 2255-2259.

66. Kou, R.; Shao, Y.; Wang, D.; Engelhard, M.H.; Kwak, J.H.; Wang, J.; Viswanathan, V.V.; Wang, C.; Lin, Y.; Wang, Y.; Aksay, I.A.; Liu, J. Enhanced activity and stability of Pt catalysts on functionalized graphene sheets for electrocatalytic oxygen reduction. Elctrochem. Commun. 2009, 11, 954-957.

67. Seger, B.; Kamat, P.V. Electrocatalytically active graphene-platinum naocomposites. Role of 2-D carbon support in PEM fuel cell. J. Phys. Chem. C 2009, 113, 7990-7995.

68. Seo, M.H.; Choi, S.M.; Kim, H.J.; Kim, W.B. The graphene-supported Pd and Pt catalysts for highly active oxygen reduction reaction in an alkaline condition. Electrochem. Commun. 2011, 13, 182-185.

69. Bong, S.; Kim, Y.R.; Kim, I.; Wooa, S.; Uhmb, S.; Lee, J.; Kim, H. Graphene supported electrocatalysts for methanol oxidation. Electrochem. Commun. 2010, 12, 129-131.

70. Dong, L.; Gari, R.R.S.; Li, Z.; Graig, M.M.; Hou, S. Graphene-supported platinum-ruthenium nanoparticles with high electrocatalytic activity for methanol ethanol oxidation. Carbon 2010, 48, 781-787.

71. Liu, X.; Wang C.Z.; Yao, Y.X.; Lu, W.C.; Hupalo, M.; Tringides, M.C.; Ho, K.M. Bonding and charge transfer by metal adatom adsorption on graphene. Phys. Rev. B 2011, 83, 235411:1-235411:12.

72. Liu, X.; Wang, C.Z.; Hupalo, M.; Yao, Y.X.; Tringides, M.C.; Lu, W.C.; Ho, K.M. Adsorption and growth morphology of rare earth metals on graphene studied by ab initio calculations and scanning tunneling microscopy. Phys. Rev. B 2010, 82, 245408:1-245408:7.

73. Hupalo, M.; Liu, X.; Wang, C.Z.; Lu, W.C.; Yao, Y.X.; Ho, K.M.; Tringides, M.C. Metal nanostructure formation on graphene: Weak versus strong bonding. Adv. Mater. 2011, 23, 2082-2087.

74. Hupalo, M.; Binz, S.; Tringides, M.C. Strong metal adatom substrate interaction of Gd and Fe with graphene. J. Phys. Condens. Matter 2011, 23, 045005:1-045005:7.

75. Liu, X.; Wang, C.Z.; Hupalo, M.; Lu, W.C.; Thiel, P.A.; Ho, K.M.; Tringides, M.C. Fe-Fe adatom interaction and growth morphology on graphene. Phys. Rev. B 2011, 84, 235446:1-235446:5. 
76. Liu, X.; Wang, C.Z.; Hupalo, M.; Lu, W.C.; Tringides, M.C.; Yao, Y.X.; Ho, K.M. Metals on graphene: Correlation between adatom adsorption behavior and growth morphology. Phys. Chem. Chem. Phys. 2012, 14, 9157-9166.

77. Binz, S.M.; Hupalo, M.; Liu, X.; Wang, C.Z.; Lu, W.C.; Thiel, P.A.; Ho, K.M.; Conrad, E.H.; Tringides, M.C. High Island Densities and Long Range Repulsive Interactions: Fe on Epitaxial Graphene. Phys. Rev. Lett. 2012, 109, 026103:1-026103:5.

78. Liu, X.; Hupalo, M.; Wang, C.Z.; Lu, W.C.; Thiel, P.A.; Ho, K.M.; Tringides, M.C. Growth morphology and thermal stability of metal islands on graphene. Phys. Rev. B 2012, 86, 081414:1-081414:5.

79. Coraux, J.; N'Diaye, A.T.; Engler, M.; Busse, C.; Wall, D.; Buckanie, N.; Heringdorf, F.-J.M.; Gastel, R.; Poelsema, B.; Michely, T. Growth of graphene on $\operatorname{Ir}(111)$. New J. Phys. 2009, 11, 023006:1-023006:22.

80. Coraux, J.; N'Diaye, A.T.; Busse, C.; Michely, T. Structural coherency of graphene on $\operatorname{Ir}(111)$. Nano Lett. 2008, 8, 565-570.

81. N'Diaye, A.T.; Coraux, J.; Plasa T.N.; Busse, C.; Michely, T. Structure of epitaxial graphene on Ir(111). New J. Phys. 2008, 10, 043033:1-043033:16.

82. Bartelt, N.C.; McCarty, K.F. Graphene growth on metal surface. MRS Bull. 2012, 37, 1158-1165.

83. Wintterlin, J.; Bocquet, M.-L. Graphene on metal surfaces. Surf. Sci. 2009, 603, 1841-1852.

84. Giovannetti, G.; Khomyakov, P.A.; Brocks, G.; Karpan, V.M.; van den Brink, J.; Kelly, P.J. Doping graphene with metal contacts. Phys. Rev. Lett. 2008, 101, 026803:1-026803:4.

85. Dedkov Y.S.; Fonin, M.; Rüdiger, U.; Laubschat, C. Rashba effect in the graphene/Ni(111) system. Phys. Rev. Lett. 2008, 100, 107602:1-107602:4.

86. Nie, S.; Wofford, J.M.; Bartelt, N.C.; Dubon, O.D.; McCarty, K.F. Origin of the mosaicity in graphene grown on $\mathrm{Cu}(111)$. Phys. Rev. B 2011, 84, 155425:1-155425:7.

87. Varykhalov, A.; Sánchez-Barriga, J.; Shikin, A.M.; Biswas, C.; Vescovo, E.; Rybkin, A.; Marchenko, D.; Rader, O. Electronic and magnetic properties of quasifreestanding graphene on Ni. Phys. Rev. Lett. 2008, 101, 157601:1-157601:4.

88. Wofford, J.M.; Starodub, E.; Walter, A.L.; Nie, S.; Bostwick, A.; Bartelt, N.C.; Thürmer, K.; Rotenberg, E.; McCarty, K.F.; Dubon, O.D. Extraordinary epitaxial alignment of graphene islands on Au(111). New J. Phys. 2012, 14, 053008:1-053008:10.

89. Vázquez de Parga, A.L.; Calleja, F.; Borca, B.; Passeggi, M.C.G., Jr.; Hinarejos, J.J.; Guinea, F.; Miranda, R. Periodically rippled graphene: Growth and spatially resolved electronic structure. Phys. Rev. Lett. 2008, 100, 056807:1-056807:4.

90. Wong, S.L.; Huang, H.; Chen, W.; Wee, A.T.S. STM studies of epitaxial graphene. MRS Bull. 2012, 37, 1195-1202.

91. Hupalo, M.; Conrad, E.H.; Tringides, M.C. Growth mechanism for epitaxial graphene on vicinal 6H-SiC(0001) surface: A scanning tunneling microscopy study. Phys. Rev. B 2009, 80, 041401:1-041401:4.

92. Lu, W.; Boeckl, J.J.; Mitchel, W.C. A critical review of growth of low-dimensional carbon nanostructures on $\mathrm{SiC}(0001)$ : Impact of growth environment. J. Phys. D 2010, 43, doi:10.1088/0022-32-3727/43/37/374004. 
93. Al-Temimy, A.; Riedl, C.; Starke, U. Low temperature growth of epitaxial graphene on SiC induced by carbon evaporation. Appl. Phys. Lett. 2009, 95, 231907:1-231907:3.

94. Park, J.H.; Mitchel, W.C.; Grazulis, L.; Eyink, K.; Smith, H.E.; Hoelscher, J.E. Role of extended defected $\mathrm{SiC}$ interface layer on the growth of epitaxial graphene on SiC. Carbon 2011, 49, 631-635.

95. Hass, J.; de Heer, W.A.; Conrad, E.H. The growth and morphology of epitaxial multilayer graphene. J. Phys. Condens. Matter 2008, 20, doi:10.1088/0953-83-8984/20/32/323202.

96. Gierz, I.; Riedl, C.; Starke, U.; Ast, C.R.; Kern, K. Atomic hole doping of graphene. Nano Lett. 2008, 8, 4603-4607.

97. McChesney, J.L.; Bostwick, A.; Ohta, T.; Seyller, T.; Horn, K.; González, J.; Rotenberg, E. Extended van hove singularity and superconducting instability in doped graphene. Phys. Rev. Lett. 2010, 104, 136803:1-136803:4.

98. Gierz, I.; Suzuki, T.; Weitz, R.T.; Lee, D.S.; Krauss, B.; Riedl, C.; Starke, U.; Höchst, H.; Smet, J.H.; Ast, C.R.; Kern, K. Electronic decoupling of an epitaxial graphene monolayer by gold intercalation. Phys. Rev. B 2010, 81, 235408:1-235408:6.

99. Sandin, A.; Jayasekera, T.; Rowe, J.E.; Kim, K.W.; Nardelli, M.B.; Dougherty, D.B. Multiple coexisting intercalation structures of sodium in epitaxial graphene-SiC interfaces. Phys. Rev. B 2012, 85, 125410:1-125410:5.

100. Evans, J.W.; Thiel, P.A.; Bartelt, M.C. Morphological evolution during epitaxial thin film growth: Formation of 2D islands and 3D mounds. Surf. Sci. Rep. 2006, 61, 1-128.

101. Venables, J.A.; Spiller, G.D.T.; Hanbucken, M. Nucleation and growth of thin films. Rep. Prog. Phys. 1984, 47, 399-459.

102. Stroscio, J.A.; Pierce, D.T.; Dragoset, R.A. Homoepitaxial growth of iron and a real space view of reflection-high-energy-electron diffraction. Phys. Rev. Lett. 1993, 70, 3615-3618.

103. Hupalo, M.; Tringides, M.C. Ultrafast kinetics in $\mathrm{Pb} / \mathrm{Si}(111)$ from the collective spreading of the wetting layer. Phys. Rev. B 2007, 75, 235443:1-235443:7.

104. Chan, T.L.; Yao, Y.X.; Wang, C.Z.; Lu, W.C.; Li, J.; Qian, X.F.; Yip, S.; Ho, K.M. Highly localized quasiatomic minimal basis orbitals for Mo from ab initio calculations. Phys. Rev. B 2007, 76, 205119:1-205119:10.

105. Qian, X.F.; Li, J.; Qi, L.; Wang, C.Z.; Chan, T.L.; Yao, Y.X.; Ho, K.M.; Yip, S. Quasiatomic orbitals for $a b$ initio tight-binding analysis. Phys. Rev. B 2008, 78, 245112:1-245112:22.

106. Yao, Y.X.; Wang, C.Z.; Ho, K.M. Chemical bonding analysis for solid-state systems using intrinsic oriented quasiatomic minimal-basis-set orbitals. Phys. Rev. B 2010, 81, 235119:1-235119:6.

107. Mayer, I. Charge, bond order and valence in the $a b$ initio SCF theory. Chem. Phys. Lett. 1983, 97, 270-274.

108. Shytov, A.V.; Abanin, D.A.; Levitov, L.S. Long-Range interaction between adatoms in graphene. Phys. Rev. Lett. 2009, 103, 016806:1-016806:4.

109. Bena, C. Effect of a single localized impurity on the local density of sates in monolayer and bilyaer graphene. Phys. Rev. Lett. 2008, 100, 076601:1-076601:4. 
110. Bacsi, A.; Virosztek, A. Local density of states and Friedel oscillations in graphene. Phys. Rev. $B$ 2010, 82, 193405:1-193405:4.

111. Cheianov, V.V.; Syljuåsen, O.; Altshuler, B.L.; Falko, V.I. Ordered states of adatoms on graphene. Phys. Rev. B 2009, 80, 233409:1-233409:4.

112. Saremi, S. RKKY in half-filled bipartite lattices: Graphene as an example. Phys. Rev. B 2007, 76, 184430:1-184430:6.

113. Cheianov, V.V.; Falko, V.I. Friedel oscillations, impurity scattering, and temperature dependence of resistivity in graphene. Phys. Rev. Lett. 2006, 97, 226801:1-226801:4.

(C) 2013 by the authors; licensee MDPI, Basel, Switzerland. This article is an open access article distributed under the terms and conditions of the Creative Commons Attribution license (http://creativecommons.org/licenses/by/3.0/). 